June 1996- UCLA/96/TEP/20

\title{
Cosmology and Astroparticles ${ }^{1}$
}

\author{
Graciela B. Gelmini \\ University of California, Los Angeles \\ Department of Physics and Astronomy \\ Los Angeles, California
}

\begin{abstract}
These lectures are devoted to elementary particle physicists and assume the reader has very little or no knowledge of cosmology and astrophysics. After a brief historical introduction to the development of modern cosmology and astro-particles in which the Hot Big Bang model is defined, the Robertson-Walker metric and the dynamics of the Friedmann-Robertson-Walker cosmology are discussed in section 2 . In section 3 the main observational features of the Universe are reviewed, including a description of our neighbourhood, homogeneity and isotropy, the cosmic background radiation, the expansion, the age and the matter content of the Universe. A brief account of the thermal history of the Universe follows in section 4, and relic abundances are discussed in section 5 . Section 6 is devoted to primordial nucleosynthesis, section 7 to structure formation in the Universe and section 8 to the possibility of detection of the dark matter in the halo of our galaxy. In the relevant sections recent developments are included, such as several so called "crisis" (the age crisis, the cluster baryon crisis and the nucleosynthesis crisis), and the MACHO events that may constitute the first detection of dark matter in the halo of our galaxy.
\end{abstract}

\section{Historical Introduction}

Cosmology, from the greek $\kappa o \sigma \mu o \varsigma$, that means "order", is the study of the origin and evolution of the Universe. The initial point of modern cosmology may be taken to be 1905 with the advent of Einstein's Relativity Theory, extended to General Relativity in 1915. The first solutions to Einstein's equation for a homogeneous and isotropic but time evolving Universe were found by Friedman in 1929 and in 19351936 Robertson and Walker showed that Friedman's metric could be derived from homogeneity and isotropy alone, independently of General Relativity. Friedman's equations show that a Universe containing matter and radiation is expanding (or contracting). In 1929 Hubble found the first indication of the recession of faraway galaxies with a velocity $v$ proportional to its distance $d, v=H_{o} d$, where $H_{o}$ is

1) Talks given at the V Taller de Partículas y Campos (V-TPyC) and V Taller Latinoam. de Fenomenología de las Interac. Fundam. (V-TLFIF), Puebla, México, 10/30 - 11/3 1995. 
called the Hubble constant, the present value of the Hubble parameter (constant in space but not in time). Notice that the subscript zero indicates a present value in cosmology.

Together with the belief that we are not in a special place in the Universe, elevated to a principle in modern cosmology, the Cosmological Principle, the recession from us of distant galaxies means that the Universe is expanding (so that any two bodies not gravitationally bound to each other are receding from each other). If the Universe was smaller in the past and it expanded adiabaticlly, it was denser and hotter. Gamow was first in taking seriously this idea. He proposed in 1946 that all the matter in the Universe was hot and reached nuclear and larger densities in the past. This is the "Hot Big Bang" model [1]. This model implies a beginning of the Universe a time $t_{o} \simeq H_{o}^{-1}$ ago. Hubble's estimate of $H_{o}$, the Hubble constant, was around $5000 \mathrm{~km} /(\mathrm{sec} \mathrm{Mpc})$, larger than the presently known value of 40-100 $\mathrm{km} /(\mathrm{sec} \mathrm{Mpc})$, what yielded a lifetime of the Universe $t_{o} \simeq H_{o}^{-1} \simeq 4.5 \times 10^{9} \mathrm{yr}$, shorter than the already known age of the Earth $(1 \mathrm{pc}=1$ parsec $=3.2615$ light-yr $)$.

In part because the measured $t_{o}$ was too short at the time, in part due to the philosophical difficulty of accepting that the Universe had a beginning, the competing "Steady-State Universe" model was introduced by Bond, Gold and Hoyle in 1948. In this model the Universe is homogeneous also in time, it always looked as it looks now. Because in the Hot Big Bang model matter and radiation were in equilibrium at early times, a remanent of radiation with a black body spectrum is expected (that would not be there in a Steady-State Universe), as predicted by Alpher and Herman [2] in 1949. The observation of this radiation by Penzias and Wilson [3] (as explained by Dicke et al. [4]) in 1965, confirmed the Hot Big Bang model. It is remarkable that Alpher and Herman predicted the temperature of this cosmic microwave background radiation, CMBR, to be $5^{\circ} \mathrm{K}$, quite close to its actual temperature of $2.7^{\circ} \mathrm{K}$. Actually, the experimental confirmation of the Hot Big Bang already existed when Gamow first proposed it, in 1946. Astronomers knew since 1941 that interstellar CN molecules are excited in high rotational levels, as seen through $\mathrm{CN}$ absorption lines in the spectrum of stars, indicating that those molecules are in a thermal bath at about $3^{\circ} \mathrm{K}$.

Gamow's main interest in proposing the Hot Big Bang was the primordial synthesis of nuclei. It was soon realized that the synthesis of heavy elements had to be done in stars, what lead to the study of stellar evolution. Only in 1966 the quantitative study of primordial nucleosynthesis started, the synthesis of ${ }^{4} \mathrm{He}$ at first and that of $\mathrm{D},{ }^{3} \mathrm{He}$ and ${ }^{7} \mathrm{Li}$ only later, in the early 70 's.

This period, the early 70's, can be considered as the beginning of astro-particles. In 1967 (the year when the standard electroweak model was proposed) Sakharov noted the essential elements necessary to generate the asymmetry between matter and antimatter in our Universe, namely the generation of baryon number or baryogenesis. One of them is the violation of baryon number, that encountered in Grand Unified Theories (first proposed by Georgi and Glashow in 1974) its most natural source. In the 70's cosmological and astrophysical implications of neutrinos were first studied, leading to the battery of tests that limit the number of neutrino 
species, their mass, cosmological density, lifetime, decay modes etc. Also, it was in 1975 when the first evidences for the dark matter were discussed and the idea of inflation came about in 1980. (For references and more details about the content of this section so far, see e.g. [5])

The last fifteen years can be considered to be the golden age of astro-particles, in which the connection of particle physics and cosmology has lead to a number of important ideas. Among them is the idea of non-baryonic dark matter -see section 8-, that is explored in simulations of galaxy formation confronted with observational cosmological data and in experimental dark matters searches, both with cryogenic detectors and large neutrino detectors, supplemented by accelerator bounds. Other examples are the models for baryogenesis and for inflation, the interplay between the properties of proposed particles and their cosmological and astrophysical consequences, the introduction of particle physics experimental techniques to study astrophysical problems (such as in large "neutrino telescopes" or in the search for MACHOS -see section 8- in the halo of our galaxy). As an example of this last point, let us mention that experiments designed to search for the decay of protons predicted by Grand Unified Theories (IMB and Kamiokande) observed for the first time neutrinos from a supernova, SN 1987A, inaugurating with this observation of neutrinos from outside the solar system, what we can call neutrino-astronomy.

\section{The Hot Big Bang Model}

2.1 The Model - The Hot Big Bang (Hot BB), the standard model of cosmology, establishes that the Universe is homogeneous, isotropic (thus, it is a FriedmannRobertson-Walker Universe) and expanding from a state of extremely high temperature and density. Thus the early Universe can be described as an adiabaticlly expanding classical gas of relativistic particles, namely radiation, in local thermal equilibrium at a temperature $T$, that changes with time, $T(t)$. The lifetime of the Universe $t$ is counted from the moment the expansion started, taken to be $t=0$.

This model is based on General Relativity, the Cosmological Principle and three major empirical pieces of evidence, namely the Hubble expansion, the cosmic blackbody microwave background radiation (CMBR) and the relative cosmic abundance of the light elements (up to ${ }^{7} \mathrm{Li}$ ). Because the model is based on General Relativity, it is for sure not valid in the realm of Quantum Gravity, $T>M_{\mathrm{Plank}}=1.22 \times 10^{19}$ $\mathrm{GeV}$ and $t<10^{-43}$ sec. The Cosmological Principle postulates that we do not live in a special place in the Universe, by requiring that every comoving observer in the "cosmic fluid" has the same history. The "cosmic fluid" has as particles clusters of galaxies, and "comoving" in practice means at rest with the galaxies within a 100 Mpc radius (one parsec, $1 \mathrm{pc}=3.26$ light-years $=3.09 \times 10^{18} \mathrm{~cm}$ ), as will become clear below (see section 3.2). The Hubble parameter $H$ provides the proportionality between the velocity $v$ of recession of faraway objects, and their relative distance $d$,

$$
v=H d .
$$


The CMBR was produced at $\mathrm{t}_{\mathrm{rec}} \simeq 3 \times 10^{5} \mathrm{y}$, the recombination epoch, when atoms became stable. It has a blackbody spectrum and it is remarkably isotropic. Finally, the earliest available proof of the consistency of the Hot BB model is provided by the cosmological abundance of ${ }^{4} \mathrm{He}$ and of the trace elements $\mathrm{D},{ }^{3} \mathrm{He}$ and ${ }^{7} \mathrm{Li}$. Their abundances, differing by several orders of magnitude, are well accounted for in terms of nuclear reactions that occur at $t_{\mathrm{BBN}} \simeq 10^{-2}-10^{2} \mathrm{sec}$, the Big Bang nucleosynthesis $(\mathrm{BBN})$ epoch, when the photon temperature was $T_{\mathrm{BBN}} \simeq 10-0.1$ $\mathrm{MeV}$ (necessarily below the binding energy of the light nuclei). Let us expand on these points.

2.2 Friedman-Robertson-Walker Models Homogeneity and isotropy restrict the space-time metric to be of the Friedmann-Robertson-Walker form,

$$
d s^{2}=d t^{2}-a^{2}(t)\left[\frac{d r^{2}}{1-k r^{2}}+r^{2}\left(d \theta^{2}+\sin ^{2} \theta d \phi^{2}\right)\right] .
$$

This metric depends on a global scale factor $a(t)$ that describes the overall expansion of the Universe, i.e. a physical distance $\lambda$ increases with the Hubble expansion as $\lambda=a(t) \lambda_{c}$, where $\lambda_{c}$ is the constant distance measured in a comoving reference frame (a frame that follows the Hubble expansion, like a grid painted on an expanding balloon), whose coordinates are $r, \theta$ and $\phi$. So $\lambda_{c}, r, \theta, \phi$ do not change with the expansion of the Universe. With the usual choice of $a_{o}=1$ for the present value of the scale factor, $\lambda_{c}$ corresponds to the present physical distance. The time coordinate $t$ in Eq. (2) is the proper time measured by a comoving observer, an observer (located at fixed $r, \theta$ and $\phi$ ) that moves with the local Hubble flow. $k$ is the curvature parameter. The spatial curvature, ${ }^{3} K(t) \equiv k / a(t)^{2}$, can be either positive (corresponding to a closed Universe with the geometry of a 3 -sphere), or zero (corresponding to a flat open Universe), or negative (corresponding to an open Universe with the geometry of a 3-hyperboloid) depending on the value of the curvature factor. Through rescaling of the coordinates, $k$ can be chosen to be $+1,0$ or -1 respectively.

This metric yields the Hubble expansion if the Hubble parameter $H(t)$, defined as $H(t) \equiv \dot{a}(t) / a(t)$, is positive, since taking $t, \theta$, and $\phi$ constant, $d s=d D(t)$ where $D(t)$ is the proper distance, $D(t) \equiv a(t) \int_{o}^{r} d r\left(1-k r^{2}\right)^{-1 / 2}$, so that the proper velocity $V \equiv d D(t) / d t$ follows Hubble's law, $V \equiv H D$. However $V$ and $D$ are not operational quantities (those that can be measured) such as the velocity of recession $v$ and the luminosity distance $d_{L}$ (see, for example, [5]). $v$ is measured through the red-shift $z$ of the observed light (of wavelength $\lambda_{o}$ ) with respect of the emitted light (of wavelength $\lambda_{e}$ ), $1+z \equiv \lambda_{o} / \lambda_{e}=a\left(t_{o}\right) / a\left(t_{e}\right) \simeq v / c$, where $c$ is the velocity of light. This is the non-relativistic expression, only valid for $z \ll 1$, otherwise $(v / c)=\left[(1+z)^{2}-1\right]\left[(1+z)^{2}+1\right]^{-1} \cdot d_{L}$ is measured through the absolute luminosity $\mathcal{L}$ of the emitting body (whose difficult determination is the origin of the uncertainties in the measurements of $H$ ) and the measured flux $\mathcal{F}, d_{L}^{2} \equiv \mathcal{L} /$ $4 \pi \mathcal{F}$. Thus Hubble's law

$$
H_{o} d_{L} \simeq z+\frac{1}{2}\left(1-q_{o}\right) z^{2}+\cdots
$$


reduces to Eq.(1) with $d=d_{L}$, for $v \ll c$. The deceleration parameter $q_{o} \equiv$ $-\left(\ddot{a} a / \dot{a}^{2}\right)_{o}$, if not zero, becomes important at moderate $z$, where yet poorly known changes in the sources due to their evolution also become important. For this reason $q_{o}$ is only known to be at most of order one. $H_{o}$, the Hubble constant, the present value of the Hubble parameter $H$, is at present observationally determined within a factor of 2 to be

$$
H_{o}=100 h \frac{\mathrm{km}}{\sec \mathrm{Mpc}},
$$

with $h=0.4-1$. Through Hubble's equation, recession velocities or red-shifts are translated into distances,

$$
d_{L} \simeq 10^{-2} \frac{c z}{\mathrm{~km} / \mathrm{sec}} h^{-1} \mathrm{Mpc}=3000 z h^{-1} \mathrm{Mpc}
$$

for $z<1$. Notice that the units of distance are $\left(h^{-1} \mathrm{Mpc}\right)$ in this case.

2.3 Evolution of the Universe - The dynamics of the global scale factor $a(t)$ is determined by the content of the Universe (in matter, radiation and vacuum energy) through the Einstein field equations, $R_{\mu \nu}=\frac{1}{2} R g_{\mu \nu}=8 \pi G T_{\mu \nu}-\Lambda g_{\mu \nu}$. Spatial homogeneity and isotropy require the total stress-energy tensor $T_{\mu \nu}$ to be diagonal and with equal space components. The simplest realization corresponds to a perfect fluid $T_{\mu \nu}=\operatorname{diag}(\rho(t),-p(t),-p(t),-p(t))$ where $\rho(t)$ and $p(t)$ are the energy density and the pressure respectively. $\Lambda$, the cosmological constant introduced by Einstein in 1917 in order to obtain a static Universe (idea rejected experimentally by the discovery of the Hubble expansion in 1929), is associated in modern terms with vacuum energy. It can be incorporated into $T_{\mu \nu}$ by adding $\rho_{\mathrm{vac}}=\Lambda / 8 \pi G$ and $p_{\mathrm{vac}}=-\rho_{\mathrm{vac}}$ to the $\rho$ and $p$ of radiation and matter to obtain $\rho_{\text {total }}=\rho+\rho_{\text {vac }}$ and $p_{\text {total }}=p+p_{\text {vac }}$. With this $T_{\mu \nu}$ there are only two independent Einstein equations, from the 00 and $i i$ components respectively. From $\mu=0, \nu=0$ we obtain the Friedmann equation,

$$
H^{2}=\left(\frac{\dot{a}}{a}\right)^{2}=\frac{8 \pi G}{3} \rho-\frac{k}{a^{2}}+\frac{\Lambda}{3}
$$

whose Newtonian meaning of energy conservation for a unit mass test particle can be seen (for $\Lambda=0$ ) by writing it as $\dot{a}^{2} / 2-G M / a=-k / 2$ for $M=(4 / 3) \pi a^{3} \rho$, where the total energy is $-k / 2$. Combining the 00 and $i i$ components one gets

$$
\frac{\ddot{a}}{a}=\frac{-4 \pi G}{3}(\rho+3 p)+\frac{\Lambda}{3},
$$

that for matter (gas, dust), for which $p=0$ (and $\Lambda=0$ ), has the meaning of acceleration equal force over mass, $\ddot{a}=-G M / a^{2}$.

In order to determine the three unknown function of time in these equations, $a(t)$, $\rho_{\text {total }}(t)$ and $p_{\text {total }}(t)$ we need a third independent equation. This is the equation 
of state, $p=p(\rho)$ provided by thermodynamics. For only relativistic particles and radiation, $p_{R}=\rho_{R} c^{2} / 3$, while for only matter (gas, dust) $p_{M}=0$ (for a gas $p_{M}=n k T \sim \rho_{M} v^{2} \ll \rho c^{2}$, where $n$ is the number density and $k$ is the Boltzmann constant). It is convenient to introduce the energy conservation equation, $T_{, \nu}^{\mu \nu}=0$ for $\mu=0$, that is not independent of Eqs. (6) and (7), $\dot{\rho}=-3(\dot{a} / a)(\rho+p)$. Using the equations of state in this equation, one obtains $\rho$ as a function of $a, \rho_{R} \sim a^{-4}$ and $\rho_{M} \sim a^{-3}$, and using these in Eq. (6) one obtains,

$$
a_{R} \sim t^{1 / 2}, \quad H_{R}=\frac{1}{2 t}, \quad a_{M} \sim t^{2 / 3}, \quad H_{M}=\frac{2}{3 t} .
$$

Thus, given a certain value of $H_{o}$, a Universe radiation dominated for most of its life is younger than a matter dominated one. The relations in Eq. (8) can be easily obtained by using $a \sim T^{-1}$ (this is the entropy conservation condition, derived from the energy conservation equation in thermal equilibrium). Then, we know that $t^{-1} \sim H \sim \sqrt{\rho}$ (from the Friedmann Eq. (6)) and we know that $\rho_{R} \sim T^{4}$ and $\rho_{M} \sim m / a^{3} \sim T^{3}$, therefore $t \sim T^{-2} \sim a^{2}$ for radiation and $t \sim T^{-3 / 2} \sim a^{3 / 2}$.

With only vacuum, namely with $p=\rho=0$ and $\Lambda>0$ constant, neglecting the curvature term we obtain from Eq. (6) an exponential expansion of the scale factor $a \sim e^{H T}$ with $H=\Lambda / \sqrt{3}$. Notice that with an exponential expansion the curvature term becomes negligibly very fast, $k / a^{2} \ll \Lambda / 3$. This is precisely why a period of vacuum dominated expansion in the early Universe, called "inflation", has been proposed to explain the flatness (see section 3.7) of the Universe.

Inflation would happen during a period of supercooling of the Universe (so that the density of radiation and matter, proportional to $T^{\alpha}, \alpha=4,3$ become negligible) while the Universe is rolling down the almost constant potential $V(\phi) \simeq V_{o}$ of a scalar field, the "inflaton" $\phi$, so that $\rho_{\mathrm{vac}} \simeq V_{o} \gg \rho_{R}, \rho_{M}$. At the end of inflation it is necessary to convert $V_{o}$ into radiation and matter, through the decay of the $\phi$ field, in a process called "reheating", that generates the Universe as we know it, still with a negligible curvature. We can rewrite Eq. (6) in the form

$$
\frac{k}{H^{2} a^{2}}=\frac{\rho_{\text {total }}}{\left(3 H^{2} / 8 \pi G\right)}-1 \equiv \frac{\rho_{\text {total }}}{\rho_{c}}-1 \equiv \Omega-1
$$

where $\Omega$ is the density in units of the critical density, $\rho_{c} \equiv 3 H^{2} /(8 \pi G)=$ $10.5 h^{2}\left(\mathrm{keV} / \mathrm{cm}^{3}\right)=1.8810^{-29} h^{2}\left(\mathrm{~g} / \mathrm{cm}^{3}\right)$. Notice that spatially open, flat and closed Universes (with $k<0, k=0$ and $k>0$ respectively) correspond to $\Omega<1$, $\Omega=1$ and $\Omega>1$ respectively. So, a long enough period of inflation produces $a^{-2} \rightarrow 0$, thus $1-\Omega^{-1} \sim\left(k / a^{2} \rho_{\text {total }}\right) \simeq 0$ (since $\rho_{\text {total }}=\rho_{\text {vac }}$ is constant $)$. That is, $\Omega=1$ after a long enough inflation.

Notice that only for $\Lambda=0$ there is a correspondence between spatial geometry and the future evolution of the Universe, at $t \rightarrow \infty$. Let us call here $\Omega_{o}$ the density of matter and radiation, $\Omega_{o}=\Omega_{R}+\Omega_{M}$, so that the total density is $\Omega=\Omega_{o}+\Omega_{\Lambda}$. Because for matter and radiation $\rho \sim a^{-\alpha}$ with $\alpha=3$ or 4 , for large values of the scale factor $a$ the curvature factor $k / a^{2}$ dominates the r.h.s of Eq. (6). In this 
case, an open or flat Universe, i.e. with $\Omega=\Omega_{o} \leq 1(k \leq 0)$, expands forever and a closed Universe, with $\Omega=\Omega_{o}>1(k>0)$ recollapses. A cosmological constant $\Lambda$ dominates instead the r.h.s. of Eq. (6) for large values of $a$, and for $\Lambda>0$ the Universe expands exponentially forever at large times (even if $\Omega \geq 1$ ), as can be seen in Fig. 1 (taken from [6]).

Notice that for matter or radiation $\rho+3 p>0$, what, through the Eq. (7) (with $\Lambda=0$ ), implies that $\ddot{a}<0$, so the expansion of the Universe is slowed down by the gravitational pulling of the mass content of the Universe, and consequently $t<H^{-1}$ (since $t=H^{-1}$ corresponds to $\ddot{a}=0$ ). On the other hand, for a vacuum dominated Universe the pressure is negative, $p_{\mathrm{vac}}=-\rho_{\mathrm{vac}}$ and Eq. (7) shows that gravity is repulsive, $\ddot{a}=\Lambda a / 3>0$ and consequently $t>H^{-1}$. Thus, a non-zero

cosmological constant yields larger values of $t_{o}$ for a given $H_{o}$ (see Fig. 1 ) than matter and radiation alone. We see, therefore, how a non-zero $\Lambda$ can resolve the present possible conflict between a large value of $H_{o}$ and a large value of $t_{o}$, the "age crisis" we will mention later (see section 3.6). (For references and more details about this section see e.g. [5])

\section{Observational Features Of The Universe}

3.1 Topography of Our Neighbourhood - Galaxies are the building blocks of our Universe. Normal galaxies have masses of $10^{8}$ to $10^{12} M_{\odot}$ (where $M_{\odot}$ is the solar mass), while dwarf galaxies have $10^{6}$ to $10^{7} M_{\odot}$ (same mass as that of globular clusters, old systems of stars spherically distributed around the center of galaxies like ours). We live in a spiral galaxy, the Milky Way, with a thin disk of a few 100

FIGURE 1. Evolution of the cosmic scale factor $a(t)$ in different FRW models. 
pc of thickness and approximately $12 \mathrm{kpc}$ of radius. The Sun is in one of its arms, at about $8 \mathrm{kpc}$ from the center.

Galaxies form gravitationally bound systems: binary systems, groups (that are systems of a few to 10 galaxies, with a typical size of $1 \mathrm{Mpc}$ ), clusters (systems with hundreds of galaxies extending from a few to $10 \mathrm{Mpc}$ ) and superclusters (with thousands of galaxies in a radius of $10-50 \mathrm{Mpc}$ ). We live in a group, the Local Group, together with another large spiral galaxy, Andromeda, at roughly $700 \mathrm{kpc}$ of the Milky Way, and several small galaxies.

At the scale of superclusters, i.e. scales of 20-100 Mpc, the Universe is better described in terms of "walls", "filaments" and "voids" [7]. There are few galaxy maps at even larger scales, i.e. scales larger than $100 \mathrm{Mpc}$, and they show smoothness at those scales. Present maps only cover a small fraction of our visible Universe, whose radius is $c t_{o} \simeq 10^{10}$ light $\mathrm{yr} \simeq 3000 \mathrm{Mpc}$.

While Hubble's law and red-shift measurements are the only tools to obtain the distance of very faraway objects, the distance to nearer ones can be estimated separately from their red-shift, what allows to measure "peculiar" velocities $v_{\text {pec }}$, i.e. velocity components due to gravitational acceleration that add to the local velocity due to the Hubble flow $H_{o} d$ to give the total velocity $v=v_{\text {pec }}+H_{o} d$. In this way it has been determined that large regions of about $100 \mathrm{Mpc}$ of our local neighbourhood are moving towards a very massive system, called the Great Attractor, with large peculiar velocities of about $600 \mathrm{~km} / \mathrm{sec}$ [8] [9].

3.2 Homogeneity and Isotropy - Although these are assumptions incorporated into the $\mathrm{BB}$ model by the Cosmological Principle, there are strong observational foundations of the homogeneity and isotropy of the Universe at scales larger than $100 \mathrm{Mpc}$.

As we have just seen, the Universe looks lumpy and large regions move with large peculiar velocities at scales $\lambda<100 \mathrm{Mpc}$, however the Universe looks smooth at larger scales and we also know it was smooth in the past. There are few 3dimensional galaxy maps that reach the necessary large scales to test homogeneity, containing in total only of the order of $10^{4}$ galaxies, but by the end of the century $10^{6}$ will be mapped. These are red-shift surveys where distances are obtained from $z$ though Hubble's law. Angular photometric galaxy surveys instead, provide 2dimensional information for several $10^{6}$ galaxies. Both types of galaxy maps show smoothness at $\lambda>100 \mathrm{Mpc}$, in the case of 2-dimensional maps by showing that angular correlations decrease steeply with increasing angular distance in the sky (see for example [6] for references). But the best evidence of homogeneity and isotropy is provided by the isotropy of the CMBR, for which measurements of the temperature in different regions of the sky show very small anisotropies, smaller than $10^{-4}$, i.e. the r.m.s. temperature fluctuation measured between two points in the sky separated by an angle $\theta$ is $(\delta T / T)_{\theta} \lesssim 10^{-5}[10]$ [11]. This shows both the smoothness of the Universe at the moment of emission of the radiation and the isotropy of the expansion since then, because the present measured wavelength of the CMBR photons now is $\lambda_{\text {now }}=a(t) \lambda_{\mathrm{e}}$, where $\lambda_{\mathrm{e}}$ is the wavelength at emission, and any space dependence of the scale factor $a(t)$ would produce anisotropies. The 
CMBR photons are emitted from the last scattering surface, that is the Universe at recombination $\left(t \simeq 3 \times 10^{5} \mathrm{yr}, T \simeq 0.3 \mathrm{eV}\right.$ and $\left.z \simeq 1100\right)$.

3.3. Cosmic Microwave Background Radiation - The best blackbody in the Universe is the Universe. The COBE (Cosmic Background Explorer) Satellite measured the CMBR spectrum in 1992 for wavelengths between 0.05 and $1 \mathrm{~cm}$, and found a blackbody with temperature $T_{o}=2.726 \pm 0.010^{\circ} \mathrm{K}$, with deviations of less than $0.03 \%$ [12]. This provides fundamental evidence for a Hot BB. As we just mentioned, the CMBR was produced at the recombination epoch, when atoms became stable and replaced ions and electrons in a plasma as the constituents of the Universe. At this moment the mean free path of photons, that was very short in the preceding plasma, became very long, so that the photons that last scattered then can reach us. Knowing so well the CMBR temperature, we know with great accuracy the number and energy density of the CMBR photons, that are the most abundant in the Universe by several orders of magnitude (see e.g. [5] p. 143), $n_{\gamma}=2 \zeta(3) T_{o}^{3} / \pi^{2}=411 / \mathrm{cm}^{3}, \rho_{\gamma}=\pi^{2} T_{o}^{4} / 15=4.71 \times 10^{-34}\left(\mathrm{~g} / \mathrm{cm}^{3}\right)$ Using $\rho_{c}$ in Eq. (9), we see that $\Omega_{\gamma} \simeq 10^{-5}$.

Anisotropies in the CMBR are expected due to our motion with respect to the CMBR rest frame, and due to the density inhomogeneities that triggered structure formation in the Universe. In fact COBE measured a dipole anisotropy corresponding to a velocity of $627 \pm 22 \mathrm{~km} / \mathrm{sec}$ of our Local Group of galaxies with respect to the CMBR rest frame (COBE even saw the rotation of the Earth around the Sun!), and measured anisotropies $(\delta T / T)_{\theta}$ for angles $\theta=7^{\circ}$ to $90^{\circ}$. At $90^{\circ} \mathrm{COBE}$ measured a quadrupole anisotropy of $(\delta T / T)_{90^{\circ}} \simeq 0.5 \times 10^{-5}$ [10]. Results from other experiments in balloons are available now at smaller angles, $\theta=0.5^{\circ}$ to $90^{\circ}$ and the results show $\delta T / T \lesssim 10^{-5}$ after subtracting the dipole.

The horizon size at recombination, $c t_{\text {rec }}$, corresponds to an angle $\theta_{H} \simeq 1^{o}(\Omega)^{-1 / 2}$ in the present sky. Larger angles correspond to causally disconnected regions at the time of emission of the CMBR photons. Thus the smallness of the anisotropies at angles $\theta \lesssim 1^{\circ}$ indicates that the Universe was smooth at recombination, and at larger angles, $\theta \gtrsim 1^{\circ}$ it indicates that the Universe is smooth on very large scales. The temperature autocorrelation function, $C(\theta)=<(\delta T(\alpha) / T) \quad(\delta T(\alpha+\theta) / T)>_{\alpha} \quad$, where $\delta T / T=(T-\bar{T}) / \bar{T}$ are temperature fluctuations with respect to the average temperature $\bar{T}=2.726^{\circ} \mathrm{K}$, is computed by measuring $\delta T / T$ at some position $\alpha$ in the sky ( $\alpha$ is given in spherical harmonics by two angles), multiplying it with $\delta T / T$ at another position $(\alpha+\theta)$ separated from the first one by an angle $\theta$ and averaging over all positions. The expansion of $C(\theta)$ in Legendre polynomials defines the multiple moments $C_{\ell}$, $C(\theta)=(4 \pi)^{-1} \sum_{\ell}(2 \ell+1) C_{\ell} P_{\ell}(\cos \theta)$, so that the anisotropy at a certain angle $\theta$ is

$$
\left(\frac{\delta T}{T}\right)_{\theta} \simeq \sqrt{\ell(\ell+1) C_{\ell}}, \quad \ell \simeq\left(200^{\circ} / \theta\right) .
$$

We see that $\ell \geq 200$ correspond to scales within the horizon at decoupling, where matter can move due to density perturbations and where the photons pick up 
additional energy due to the scattering from moving matter (mainly Thompson scattering on electrons). This gives origin to Doppler (or acoustic) peaks in the spectrum of multiple moments. The position of the first peak should happen at the scale of the horizon $\ell_{\text {peak }} \simeq 200(\Omega)^{-1}$, as shown in Fig. 2 [13] for different dark matter models (see section 7). Thus, in the future, the position of the first peak will determine $\Omega$ within a $10 \%$. The height of the peaks depends on the density in baryons, $\Omega_{B}$ and the location and relative height of the peaks will allow to discriminate different models of galaxy formation, once all $C_{\ell}$ are measured. Actually only the $C_{\ell}$ for $\ell<O\left(10^{3}\right)$ are relevant, because larger values of $\ell$ correspond to scales $\theta<8^{\circ}$ that are inside the thickness of the last scattering surface, so the information at those small scales is smeared out [11].

3.4 The Expansion of the Universe - Hubble's law in Eq.(1) is valid at $z \ll 1$. At $z \gtrsim 1$ the law becomes quadratic (see Eq. (3)) and the deceleration parameter $q_{o}$ measures the rate at which the gravitational attraction is slowing down the expansion. Actually $q_{o}=\Omega_{o} / 2-\Omega_{\Lambda}$ (for matter dominated $\Omega_{o}$ ), so for a large cosmological constant component $\Omega_{\Lambda}, q_{o}$ could be negative and the expansion could be accelerated (recall that gravitation is repulsive in a vacuum dominated Universe). Not much can be said observationally about $q_{o}$ yet. So let us concentrate on the Hubble constant $H_{o}$.

Redshifts can be measured accurately. This allows to determine the recession velocities $v$. In general they have two components $v=H_{o} d+v_{\text {pec }}$. Thus recession velocities can be attributed solely to the Hubble flow at distances large enough for the peculiar radial velocities $v_{\text {pec }}$, the velocity components due to gravitational

FIGURE 2. CMBR anisotropy power spectra predicted by four models (lines) of structure formation (see section 7). The band shows the expected experimental error in future satellite experiments. 
acceleration, to be negligible, $H_{o} d \simeq v \gg v_{\text {pec }}$, what happens at $d \gg h^{-1} \mathrm{Mpc}$, say distances larger than $50 \mathrm{Mpc}$, because $v_{\text {pec }} \simeq O(100 \mathrm{~km} / \mathrm{sec})$.

In order to measure $H_{o}=v / d$ (Eq. (1)), distances and velocities must be determined separately. The dificulty in the measurement of $H_{o}$ reside in determining $d$. Different techniques to measure distances lead to the spread of roughly a factor of two in results for $h$ (the Hubble constant in units of $100(\mathrm{~km} / \mathrm{sec} \mathrm{Mpc})$ see Eq. (4)). Most methods give large values of $h, h \simeq 0.8$. For example, a recently publicized result of the Hubble Space Telescope is $h=0.8 \pm 0.17$ [14]. This determination is based on the observation of twenty Cepheids in the Virgo cluster. Cepheids are variable stars, whose period of variability is associated with their intrinsic luminosity, thus, the apparent luminosity tells the distance to the star. Some determinations give smaller values of $h$. For example, using type $I a$ supernovae a determination of $h=0.5 \pm 0.1$ was made (see e.g. [9] for references).

The problem with a large value of $h, h>0.65$, is that it yields a too young Universe, unless $\Lambda \neq 0$ or $\Omega_{o}<1$, namely unless we live in a Universe with positive cosmological constant, or open, or a combination of the two. This goes against aesthetic beliefs in a flat, matter dominated Universe, giving origin to what some call an "age crisis" (see section 3.6 below).

3.5 The Age of the Universe - There are three main methods to determine $t_{o}$. The technique of nuclear cosmochronology, uses the relative abundance of radioactive isotopes at present and at production (through rapid neutron capture processes, $r$-processes, most probably in supernovae). Comparing the two gives $t_{o}=(10-20) \times 10^{9}$ yr $[15]$.

White dwarfs are the last stage of low-mass stars. Since they are faint they can only be seen near the solar system. There is a drop in the number of white dwarfs with luminosity smaller than $3 \times 10^{-5}$ of the solar luminosity. Combining cooling models with the assumption that this cut indicates that there are no older white dwarfs that had the time to become fainter, gives the age of the disk of our galaxy. This method gives $t_{o}=(9-10) \times 10^{9}$ yr [16].

Globular clusters are old systems of a few-million stars that formed all at the same time. The evolution of stars depends on their initial mass, the more massive have shorter lifes, and finish burning Hydrogen earlier. The age of globular cluster is determined by measuring the absolute luminosity of the stars that are finishing burning $\mathrm{H}$, using well known stellar evolution theory. The lifetimes obtained in this way are $t_{o} \simeq(13-15) \times 10^{9} \mathrm{yr}$ and it is very difficult to get to $t_{o}<11-12 \times 10^{9}$ yr [17]. Thus, $t_{o} \gtrsim 13 \mathrm{Gyr}$ is taken at present to be a reasonable lower bound of the age of the Universe and $t_{o} \gtrsim 10 \mathrm{Gyr}$ is taken to be an absolute lower bound, a minimum age already uncomfortable to accommodate globular clusters.

3.6 Age Crisis? - The parameters $t_{o}, H_{o}$ and $\Omega$ are not independent, actually $H_{o} t_{o}=1.06(h / 0.80)\left(t_{o} / 13 \times 10^{9} \mathrm{yr}\right)=f_{i}(\Omega)$, where $f_{i}$ is a function of $\Omega$ that depends on the content of the Universe (see e.g. [5]). For a flat $\Omega_{o}=1$ Universe matter dominated by the dark matter (see section 3.7) with $\Lambda=0$ (until recently the model preferred by most cosmologists due to its simplicity and aesthetic appeal), one has $H_{o} t_{o}=2 / 3$ what implies that $t_{o} \gtrsim 13$ Gyr requires $h \lesssim 0.50$ and $t_{o} \gtrsim 10$ 
Gyr requires $h \lesssim 0.65$. As mentioned above, in section 3.4, these values of $H_{o}$ are lower than most present determinations (but not all). If $h$ is actually larger than 0.65, then we live in a Universe with a non-zero cosmological constant, or open, or both.

If our Universe is spatially flat, namely $\Omega=\Omega_{o}+\Omega_{\Lambda}=1$, the frequency of gravitational lensing of quasars by nearer galaxies gives a bound of $\Omega_{\Lambda} \lesssim 0.7$ [18]. The number of lensings depends on $\Lambda$, because with $\Lambda>0$ the distance to a quasar of a given redshift is larger than for $\Lambda=0$. With $\Omega=1$ and $\Omega_{\Lambda} \leq 0.7$ one gets $H_{o} t_{o}<0.96$. In this case $t_{o}>13(10)$ Gyr requires $h<0.72(0.94)$. On the other hand if $\Lambda=0$ and $h>0.65$, we need $\Omega\left(=\Omega_{o}\right)<1$, namely we live in an open Universe. For a matter dominated Universe $H_{o} t_{o}$ increases for decreasing $\Omega_{o}$, from $2 / 3$ for $\Omega_{o}=1$ to 1 for $\Omega_{o}=0$ (namely for an empty Universe $t_{o}=H_{o}^{-1}$, since $\ddot{a}=0)$. Dark matter measurements indicate that there is at least $\Omega_{o}=0.1$ in matter, what means $H_{o} t_{o} \lesssim 0.9$. In this case (with $\Lambda=0$ ) $t_{o}>13(10)$ Gyr requires $h<0.68$ (0.88). Of course a combination of $\Lambda \neq 0$ with $\Omega<1$ would also be possible, but even less appealing.

Why does $\Lambda \neq 0$ or $\Omega<1$ seem so unappealing? There is a problem of fine tuning in both cases. A cosmological constant corresponds to a non-zero vacuum energy. We cannot explain why the vacuum energy should be zero at present, but it is even less appealing to explain why if non-zero it is so small with respect to characteristic values of the potential energy of scalar fields in particle physics. Take as an example the usual Higgs potential responsible for the spontaneous breaking of the electroweak symmetry. Take $\phi$ to be the usual Higgs doublet, then the potential energy density is $V=\lambda\left(|\phi|^{2}-v^{2}\right)^{2}$ and the hight of this potential is $V_{o}=V(\phi=0)=\lambda v^{4}=\lambda(100 \mathrm{GeV})^{4}$. In units of $\mathrm{GeV}^{4}$ the critical density is $\rho_{c}=10^{-46} \mathrm{GeV}^{4}$, so $\rho_{\mathrm{vac}}=\rho_{\Lambda} \lesssim \rho_{c}=10^{-54} \lambda^{-1} V_{o}$ !

With $\Omega \neq 1$ there is an equally important fine tuning to take into account. If $\Omega \neq 1, \Omega$ increasingly separates from 1 as $t$ increases in a Universe radiation or matter dominated. Dividing Eq. (9) by $\Omega$, we find $1-\Omega^{-1}=3 K\left(8 \pi G a^{2} \rho\right)^{-1} \equiv \chi(t)$ and $\chi(t) \sim a$ for matter $\sim a^{2}$ for radiation (see Eq.(8)). Thus, in order to have $\Omega=O(1)$ (but $\neq 1$ ), $\Omega$ should have differed from 1 in the past by an extremely small amount, at most $\chi(t) \simeq 10^{-10}$ at the nucleosynthesis epoch $(T \simeq 1 \mathrm{MeV})$ or $10^{-32}$ at the Planck time $\left(T \simeq 10^{19} \mathrm{GeV}\right)$. These small initial values of $\chi(t)$ are unappealing.

3.7 Matter Content of the Universe - The luminous mass, namely the matter associated with typical stellar populations, accounts for $\Omega_{\text {Lum }} \simeq 0.003 h^{-1}$ [19]. It is determined through luminosity measurements knowing the average mass over luminosity ratio. This is, for example, 5 times the solar ratio in the solar neighborhood and $12 h$ times the solar ratio in the core of elliptical galaxies.

However there is much more than the luminous mass in the Universe. It is by now a well established fact that on all scales larger than about $10 \mathrm{kpc}$ there is a discrepancy between the amount and distribution of the luminous mass, and the mass detected through its gravitational effects. The gravitationally dominant mass component of the Universe is "dark", i.e. it is not seen either in emission 
or absorption of any type of electromagnetic radiation. This is called dark matter (DM). The most robust evidence for the DM is given by the rotation curves $v(r)$ of spiral galaxies, i.e. the orbital velocities of stars and gas clouds orbiting a spiral galaxy at a distance $r$ from its center. These velocities remain constant outside the region where the light falls exponentially off. Since $v(r)^{2}=G M(r) / r$, the flatness of the rotation curves $v(r)$ beyond a few kpc from the center means that the mass contained within a radius $r$ grows linearly with $r, M(r) \sim r$. This indicates the existence of a quasi-spherical DM halo around each galaxy whose density falls off as $r^{-2}$ outside a core radius of a few kpc. These DM haloes amount to $\Omega_{\mathrm{DM}}=0.02-0.05$ [20] [19]. The first indications of the necessity of DM came from the study of the Coma cluster by Zwicky in 1930. Applying the virial theorem to clusters of galaxies the total amount of matter in the cluster can be inferred from the characteristic velocity and average separation of its galaxies. The DM measured in this manner is $\Omega_{\mathrm{DM}} \simeq 0.1-0.2$ [19]. A more recent method uses the gravitational lensing of very far galaxies by clusters, whose total mass can be reconstructed from the deformation of the lensed galaxies into arcs and arclets. This method also yields $\Omega_{\mathrm{DM}} \simeq 0.1-0.2[21]$. Peculiar velocities are due to gravitational accelerations due to masses nearby. Large scale peculiar velocity flows, at scales of $50 \mathrm{Mpc}$ and larger, can then be used to infer total mass distributions, what yields estimates in the range $\Omega_{\mathrm{DM}} \simeq 0.2-1[9]$.

With respect to the density in baryons (namely nuclei) $\Omega_{B}$, Big Bang nucleosynthesis (BBNS) predicts the density of light elements in agreement with observations only for $\Omega_{B} h^{2} \simeq 0.01-0.02$ (see more about nucleosynthesis in section 6 ), what for $h=0.4-1$ means $0.01 \lesssim \Omega_{B} \lesssim 0.13$. This means that all the DM in the halo of galaxies could be baryonic (in the form of macroscopic objects, such as failed stars or primordial black holes for example), but also may indicate that some of the baryons are dark $\left(\Omega_{B}>\Omega_{\text {Lum }}\right)$ if $h<1$.

The BBNS estimate of $\Omega_{B}$ combined with the measurement of a "large" amount of gas in rich clusters of galaxies has led to what some call "the x-ray cluster baryon crisis" [22] [23]. In fact, the gas in the central part (until a radius of about $1 h^{-1}$ $\mathrm{Mpc}$ ) of the cores of rich clusters is seen through the x-rays emitted by the gas in hydrostatic equilibrium and it has been recently seen that the fraction of the total mass in gas in those regions is large, $\mathrm{f}=\left(M_{\text {gas }} / M_{\text {total }}\right) \simeq(0.05-0.10) h^{-3 / 2}$. Since the clusters in question are large clusters, one can think that this ratio is representative of the baryon fraction in the Universe $\rho_{B} / \rho_{M}=\Omega_{B} / \Omega_{\mathrm{DM}}$. If so, $\Omega_{\mathrm{DM}}=\Omega_{B} f^{-1}$ and using the measured large value of $\mathrm{f}$ and the BBNS upper bound of $0.02 h^{-2}$ on $\Omega_{B}$ one gets an upper limit of $\Omega \leq(0.2-0.4) h^{-1 / 2}$ [22]. If $h>0.16$ (as all measurements confirm, see section 3.4) and the bound on $\Omega_{B}$ from BBNS is correct, this would mean that either we live in an open Universe (if $\Lambda=0$ ) or in a Universe with $\Lambda \neq 0$ (if we want it flat we need $\Omega_{\mathrm{DM}}+\Omega_{\Lambda}=1$ ) or both. 


\section{Thermal History Of The Universe}

As we have seen in sections 3.3 and 3.7, the present energy in radiation $\Omega_{\gamma} \simeq 10^{-5}$, is much smaller than that of matter $\Omega_{0} \simeq \Omega_{\mathrm{DM}} \simeq 0.1-1$. This still holds true even when adding the contribution of relativistic neutrinos, with which $\Omega_{R}\left(T_{o}\right)=$ $4 \times 10^{-5} h^{-2}\left(g_{*} / 3.36\right)$, where $g_{*}$ are effective relativistic degrees of freedom and for photons and three relativistic neutrino species, $\mathrm{g}_{*}=3.36$ (see section 5 ). Due to the different evolution with temperature of the density of matter and radiation, $\rho_{R} \sim T^{4}$ and $\rho_{M} \sim T^{3}$, the radiation was dominant in the past at $T>T_{e q}$, where $T_{e q}$ is the temperature at matter-radiation equality, $\rho_{R}\left(T_{e q}\right)=\rho_{M}\left(T_{e q}\right)$, $T_{e q} \simeq 5.8 \mathrm{eV} \Omega_{o} H_{o}^{2}\left(3.36 / g_{*}\left(T_{e q}\right)\right)[5]$.

At even earlier times, particles of mass $m$ could be produced and formed part of the "primordial soup" together with photons when $T>m$. When the temperature, that is the characteristic energy of the photons, was larger than the binding energy of a certain system, this system could not survive as such. This happened with atoms for $T>1 \mathrm{eV}$ and with nuclei for $T>1 \mathrm{MeV}$. This mean that atoms became stable for the first time at the recombination epoch, $T \simeq 1 \mathrm{eV}$ and nuclei were first formed at the BBNS epoch, $T \simeq 10-0.1 \mathrm{MeV}$.

Going to still earlier times and larger $T$, QCD predicts that chiral symmetry breaking and the confinement of quarks within hadrons should happen at $T \simeq 100$ $\mathrm{MeV}$. Even earlier, the electroweak symmetry should be restored at $T>100 \mathrm{GeV}$. Still earlier, we would encounter physics beyond the standard electroweak model, whatever it might be, until the Planck scale $T \simeq 10^{19} \mathrm{GeV}$ above which a quantum theory of gravity would be required.

\section{Relic Abundances of Particles}

Let us follow the histories of particles that were in equilibrium at a high temperature $T$ in the primitive Universe. Stable particles $X$ in equilibrium have the following number densities $n_{X}$ with respect to photons $n_{\gamma}$ :

$-n_{X} / n_{\gamma}=f_{X}\left(g_{X} / 2\right), \quad$ for relativistic particles, $\left(m_{X}<<T\right)$, where $f_{X}=1$ for a boson and $3 / 4$ for a fermion, and $g_{X}$ is the multiplicity of spin states $\left(g_{X}=1\right.$ for a real spin 0 boson, $g_{X}=2$ for the photon and a Weyl or Majorana fermion, $g_{X}=3$ for a massive gauge boson, $g_{X}=4$ for a Dirac fermion);

$-n_{X} / n_{\gamma}=(\pi / 8)^{1 / 2}\left(g_{X} / 2 \zeta(3)\right)\left(m_{X} / T\right)^{3 / 2} \exp \left(-m_{X} / T\right)$, for non-relativistic particles $\left(m_{X}>>T\right)$.

Particles go out of equilibrium when their rate of interaction $\Gamma=n \sigma v$ becomes smaller than the rate at which $T$ decreases, that is the rate of expansion of the Universe, the Hubble constant $H$, since $-\dot{T} / T=\dot{a} / a=H$ (or, equivalently, when the mean interaction time $\Gamma^{-1}$ becomes larger than the age of the Universe $H^{-1}$ ). Particles for which the condition $\Gamma>H$ was never fulfilled were never in equilibrium and their number has to be computed in other ways (this is the case of axions, for 
example). Different particles go out of equilibrium at different "freeze-out" or "decoupling" temperatures $T_{\text {f.o. }}$, depending on their interactions.

Relativistic particles with weak interactions go out of equilibrium in the early Universe at $T_{\text {f.o. }} \simeq 1 \mathrm{MeV}$. The estimate of this temperature is very easy. For light left-handed neutrinos $\nu_{L}$, for temperatures smaller than the mass of the vector bosons that mediate the interaction, the $W$ and $Z,\left(M_{V} \simeq 100 \mathrm{GeV}\right)$ but larger than any other masses (of neutrinos, leptons, quarks ...) the cross section on dimensional grounds is $\sigma \simeq G_{F}^{2} T^{2}$, with $G_{F}$ the Fermi constant, $G_{F} \simeq 10^{-5} / \mathrm{GeV}^{2} \sim M_{V}^{-2}$. The number density of light particles is $n \simeq T^{3}$, and their velocity $v \simeq c=1$. On the other hand, $H \simeq \sqrt{G} T^{2}$, with $G$ the gravitational constant, $G \simeq M_{P}^{-2}$. Thus the interaction rate goes as $\Gamma \simeq n \sigma v \simeq G_{F} T^{5}$, it decreases faster with $T$ than $H$ and at $T=T_{\text {f.o. }} \Gamma$ becomes smaller than $H$. By equating $\Gamma=H$ the mentioned $T_{\text {f.o }}$ is obtained.

The number $n_{\nu}$ of light neutrinos (that are relativistic at $T \simeq 1 \mathrm{MeV}$ ) per comoving volume is kept constant after their freeze-out, but the number of photons is increased by the annihilation of $e^{+} e^{-}$. When $T$ drops below a mass threshold for the production of pairs of certain particles, $e^{+} e^{-}$in this case, these particles cease to be produced and only annihilate "heating" the photons and all the other interacting particles but not the decoupled species. Consequently, the temperature of the decoupled particles, $T_{\nu}$ in this case, becomes smaller than the photon temperature $T$. Actually, it is not that the temperature of photons increases, it just decreases at a smaller rate for a while. We can compute the ratio $T_{\nu} / T$ using entropy conservation. In fact, in thermal equilibrium the total entropy per comoving volume, $S=s a^{3}$, is conserved. Here $s$ is the entropy density $s=(p+\rho) / T$, thus $s=(4 / 3)(\rho / T)$ for radiation (and relativistic particles). Let us compute the entropy before and after $e^{+} e^{-}$annihilation and use entropy conservation, with the approximation that the scale factor $a$ before and after is the same, namely assuming there is not enough time for $a$ to change much. The entropy before the annihilation has a contribution due to $e^{+} e^{-}, s_{e}=4 \times(7 / 8) \times\left(2 \pi^{2} / 45\right) T^{3}$ and a contribution due to the photons $s_{\gamma}=2 \times\left(2 \pi^{2} / 45\right) T^{3}$. After the annihilation only the photons remain with $T=T_{\text {after }}>T_{\text {before, }}$ since $(2+7 / 2) T_{\text {before }}^{3}=2 T_{\text {after }}^{3}$. The temperature of the neutrinos is not changed (because neutrinos are decoupled) and remains equal to $T_{\text {before }}, T_{\nu}=T_{\text {before }}=(4 / 11)^{1 / 3} T$. Thus, the number of neutrinos plus anti-neutrinos, $n_{(\nu+\bar{\nu})}=n_{i}$ of every light species $\nu_{e}, \nu_{\mu}, \nu_{\tau}$, that was before $n_{i} / n_{\gamma}=(3 / 4)\left(g_{\nu} / 2\right)$ becomes $n_{i} / n_{\gamma}=(3 / 11)\left(g_{\nu} / 2\right)$, that is, $n_{i}=\left(g_{\nu} / 2\right)\left(115 / \mathrm{cm}^{3}\right)$, because the number of neutrinos even after their decoupling is still proportional to $T_{\nu}^{3}$ (see e.g. [5]).

Knowing $T_{\nu}$ we can compute the contribution of each $\nu$-species that is still relativistic to the present radiation energy density $\rho_{R}$, that is usually parametrized as $\rho_{R}=\left(\pi^{2} / 30\right) g_{*}(T) T^{4}$. Here $g_{*}(T)=\sum_{B} g_{B}\left(T_{B} / T\right)^{4}+\frac{7}{8} \sum_{F} g_{F}\left(T_{F} / T\right)^{4}$ is the effective number of relativistic degrees of freedom at the temperature $T$, the sums run over all relativistic bosons $\mathrm{B}$ and fermions $\mathrm{F}$ at $T, g_{B}$ and $g_{F}$ are the number of bosonic and fermionic degrees of freedom and $T_{B}$ and $T_{F}$ are the temperatures of each species, which are equal to $T$ only for species still in thermal equilibrium. 
For every $\nu$ species $g_{*}\left(T_{o}\right)=2 \times 7 / 8 \times\left(T_{\nu} / T_{o}\right)^{4}=2 \times 7 / 8(4 / 11)^{4 / 3}=0.454$.

Assuming all three neutrino species are still relativistic, photons and neutrinos account for $g_{*}\left(T_{o}\right)=2+3 \times 0.454=3.36$, and $\Omega_{R} h^{2}=4 \times 10^{-5}\left(g_{*} / 3.36\right)$ (as already mentioned in section 4$)$. However, it is possible that one or more neutrinos are non-relativistic at present (if $m_{\nu}>T_{o}=2.3 \times 10^{-4} \mathrm{eV}$ ), since the experimental upper bounds are $m_{\nu_{e}}<5 \mathrm{eV}, m_{\nu_{\mu}}<160 \mathrm{keV}, m_{\nu_{\tau}}<24 \mathrm{MeV}$. In this case, the contribution of the non-relativistic light neutrinos to the density of the Universe now is $\rho_{\nu}=\sum_{i=1}^{3} m_{\nu_{i}} n_{i}=\Omega_{\nu} \rho_{c}$, which means

$$
\sum_{i=1}^{3} m_{\nu_{i}}=92 \mathrm{eV} \Omega_{\nu} h^{2}\left(\frac{2}{g_{\nu}}\right) .
$$

The sum runs over all non-relativistic neutrino species lighter than $1 \mathrm{MeV}$ (because we use $n_{\nu}$ for neutrinos relativistic at freeze-out).

From Eq. (11) an upper bound on neutrino masses follows from an upper bound on the present density of the Universe, $\Omega_{\nu} \leq \Omega_{o}$. The best bound on $\Omega_{o} h^{2}$ comes from a lower bound on the lifetime of the Universe (see section 3.6). In a matter dominated Universe (with $\Lambda=0$ ), $t_{o} \gtrsim 1.3 \times 10^{10}$ yr requires $\Omega_{o} h^{2} \lesssim 0.4$, but adding the reasonable bound $h \gtrsim 0.5$ one obtains $\Omega_{o} h^{2} \lesssim 0.25$. These two bounds imply (using Eq. (11)) $\sum_{i} m_{\nu_{i}} \lesssim 37 \mathrm{eV}$ and $\sum_{i} m_{\nu_{i}} \leq 23 \mathrm{eV}$, respectively [24]. With a non-zero cosmological constant, $\Lambda>0$, these bounds are relaxed a bit, since $\Omega_{o} h^{2}$ can be larger for the same $t_{o}$. These bounds apply to the masses $m_{\nu_{i}}$ of neutrinos with two degrees of freedom, i.e. Weyl of Majorana, and full weak interactions, thus $g_{\nu}=2$. The right-handed neutrino components, even when they exist, are not counted, because either they are never in equilibrium or decoupled much earlier than the left handed components, and are therefore much less abundant.

Heavy relics $X$ of mass $m_{X}$ that become non-relativistic while still in equilibrium, have a different history. While annihilations are in equilibrium, i.e. $\Gamma_{\text {annih. }}>H$, the number of non-relativistic particles decreases with the Boltzmann factor $n_{X} / T^{3} \sim \exp \left(-m_{X} / T\right)$. When annihilations cease (because the rate $\Gamma_{\text {annih. }}$ becomes small with respect to $H), n_{X} / T^{3}$ departs from its equilibrium value and soon it becomes constant. The larger the annihilation cross-section is, the longer annihilations remain in equilibrium, and the smaller is the relic abundance $\Omega$, thus $[25]$

$$
\Omega h^{2} \simeq \frac{1 \times 10^{-37} \mathrm{~cm}^{2}}{<\sigma_{a} v>} .
$$

Here $\left\langle\sigma_{a} v\right\rangle$ is the thermal average of the non relativistic annihilation crosssection that is always proportional to $v^{-1}$, and $<>$ indicates the average over a thermal distribution of momenta (average over initial states and sum over all final states).

Eq. (12) implies that particles of mass $m_{X}$ of order $\mathrm{GeV}-\mathrm{TeV}$ with annihilation cross sections of "weak" order, i.e. $<\sigma_{\text {annih }} v>\simeq G_{F}^{2} m_{X}^{2} \simeq 10^{-37} \mathrm{~cm}^{2}\left(m_{X} / \mathrm{GeV}\right)^{2}$ 
for $m_{X}<M_{Z}$ and $\left\langle\sigma_{\text {annih }} v>\simeq \alpha / m_{X}^{2} \simeq 10^{-37} \mathrm{~cm}^{2}\left(m_{X} / \mathrm{TeV}\right)^{-2}\right.$ for $m_{X}>>M_{Z}$, have relic abundances $\Omega$ of order one, and may be, therefore, good DM candidates ( $\alpha$ is the electromagnetic constant).

Since elementary particle physicists are exploring extensions of the electroweak model, plenty of new particles with masses in the few $\mathrm{GeV}$ to a few $\mathrm{TeV}$, are hypothesized. Many of them have interactions mediated by particles in the same mass range, what means cross sections of the weak order and, thus, they can account for $\Omega \simeq 0.1$ to 1 and are good DM candidates. This is the case of the Lightest Supersymmetric Particle, for example.

In the above discussion no asymmetry between $X$-particle and antiparticle numbers was assumed. A cosmic asymmetry would insure that annihilations stop when the minoritary population is depleted, leaving many more particles than expected without an asymmetry.

\section{Primordial Nucleosynthesis, Also in Crisis?}

When the temperature of the Universe became smaller than the binding energy of nuclei, at $T \simeq 0.1 \mathrm{MeV}$, nuclei first became stable. Because neutrons are heavier than protons $n_{n} / n_{p} \sim \exp \left[-\left(m_{n}-m_{p}\right) / T\right]<1$, there are less neutrons than protons. Most of the neutrons end up into ${ }^{4} \mathrm{He}$ (approximately $25 \%$ of the mass of the Universe) together with an equal number of protons. The remaining protons stay as $\mathrm{H}$ (approximately $75 \%$ of the mass of the Universe), and trace amounts of $\mathrm{D}$, ${ }^{3} \mathrm{He}$ and ${ }^{7} \mathrm{Li}$ are produced (with $n_{\mathrm{D},{ }^{3} \mathrm{He}} / n_{\mathrm{H}} \simeq O\left(10^{-4}\right)$ and $\left(n^{7} \mathrm{Li} / n_{\mathrm{H}}\right) \simeq O\left(10^{-10}\right)$. Primordial abundances of the light-elements are difficult to infer from observations and experts differ on the relative weight they give to different measurements and methods of inference. While already in 1964 it was known that stars can only produce less then $5 \%$ of the existing ${ }^{4} \mathrm{He}$, only in the early 70 's it was shown that $\mathrm{D}$, being very weakly-bound, is easily destroyed in stars (mostly into ${ }^{3} \mathrm{He}$ ) but it cannot be produced.

The predictions of BBNS depend on the value of the baryon-to-photon ratio $\eta \equiv n_{B} / n_{\gamma}$ during the nucleosynthesis, and it is remarkable considering that the abundances are vastly different, that there is a range of $\eta \simeq O\left(10^{-10}\right)$ for which realistic abundances are obtained for all the light element. The prediction of the ${ }^{4} \mathrm{He}$ abundance also depends of the number of relativistic neutrino species in equilibrium during the BBNS, $N_{\nu}$. The abundance of ${ }^{4} \mathrm{He}$ increases with $N_{\nu}$. Thus present upper bounds on ${ }^{4} \mathrm{He}$ give an upper bound on $N_{\nu}$ of about $3 . N_{\nu}$ parametrizes also any non-standard contribution to the energy density of the Universe during the BBNS, so it would be easy to explain a BBNS prediction of $N_{\nu}$ larger than 3 . However, a bound $N_{\nu}<3$ would require at least one of the three known neutrinos to contribute less than a relativistic neutrino during BBNS. For example, a heavy neutrino could decay before the BBNS, i.e. with a lifetime $<10 \mathrm{sec}$. Otherwise, one would suspect that there is something wrong in the arguments leading to the BBNS bound on $N_{\nu}$. Precisely a bound $N_{\nu}<2.6$ (95\% C.L.), with a value $N_{\nu}=2.1 \pm$ 
0.3 ( $1 \sigma$ error), is the claim of a recent paper entitled "Big Bang Nucleosynthesis in Crisis" [26]. One of the main differences in this paper is a lower assumed range for $\mathrm{D}+{ }^{3} \mathrm{He}$. Because the abundance of ${ }^{4} \mathrm{He}$ increases with $\eta$ while that of $\mathrm{D}$ and ${ }^{3} \mathrm{He}$ decrease with $\eta\left({ }^{7} \mathrm{Li}\right.$ has a dip, but increases with $\eta$ for $\left.\eta \gtrsim 3 \times 10^{-10}\right)$ a lower assumed range for $\mathrm{D}+{ }^{3} \mathrm{He}$, pushes the acceptable range of $\eta$ in this paper, to larger values. Fig. 3 (taken from [5]) shows the dependence of the different abundance on $\eta$ and of the ${ }^{4} \mathrm{He}$ abundance on $N_{\nu}$. Because the abundance of ${ }^{4} \mathrm{He}$ also increases with $N_{\nu}$, in order to prevent getting too much ${ }^{4} \mathrm{He}$ with the larger values of $\eta$ selected by the lower D abundance, $N_{\nu}$ must be smaller. Given the dispersion in different measurements of primordial abundances (26), to claim, a "crisis" seems premature. However one should keep in mind that the primordial abundances of ${ }^{4} \mathrm{He}, \mathrm{D}+{ }^{3} \mathrm{He}$ and ${ }^{7} \mathrm{Li}$ leave a narrow range of $\eta$ where agreement with predictions for all of them is achieved and when observational uncertainties decrease further in the near future (mainly in the primordial abundance of $\mathrm{D}$ [27]) this narrow range will be further squeezed.

Let us briefly see where the different dependences with $\eta$ and $N_{\nu}$ of the various abundances shown in Fig. 3 come from (for more details see e.g. [5]). Let us start our description at $t \simeq 10^{-2} \mathrm{sec}$, when $T \simeq 10 \mathrm{MeV}, \gamma, \nu, \bar{\nu}, e^{+}, e^{-}, n$ and $p$ are in equilibrium and $n_{n} / n_{p} \simeq 0.9$ due to the difference in mass between protons and neutrons $m_{n}-m_{p}=1.293 \mathrm{MeV}$. At about $t \simeq 1 \mathrm{sec}, T \simeq 1 \mathrm{MeV}$, weak scatterings and annihilations go out of equilibrium, thus $\nu$ 's decouple and the ratio $n_{n} / n_{p}$ freezes-out at $n_{n} / n_{p} \simeq 1 / 6$ and decreases from then on only because neutrons decay (thus the BBNS predictions depend on the neutron lifetime).

The exact temperature at which the weak decoupling occurs depends on the value of Hubble parameter $H$. The larger $\mathrm{H}$, the sooner the decreasing rate of

FIGURE 3. Predicted primordial abundances as function of $\eta$ and $N_{\nu}$. 
weak scatterings and annihilations becomes equal to $\mathrm{H}$, thus the weak decoupling (or freeze-out) occurs earlier, consequently the $n_{n} / n_{p}$ ratio at freeze-out is larger and, because practically all neutrons available when nucleosynthesis finally happens end up in ${ }^{4} \mathrm{He}$, the predicted abundance of ${ }^{4} \mathrm{He}$ is also larger. Since the density of the Universe $\rho$ increases with the number of neutrino species $N_{\nu}, \mathrm{H} \sim \sqrt{\rho}$ and consequently the predicted abundance of ${ }^{4} \mathrm{He}$ also increase with increasing $N_{\nu}$.

Even if the temperature at this point, $T \simeq 1 \mathrm{MeV}$, is lower than the binding energy of ${ }^{4} \mathrm{He}$, the synthesis of this nucleus (and consequently, that of heavier nuclei) cannot happen because the deuterium $D$ has a very small binding energy, i.e. it is constantly destroyed by photons with $E>2.2 \mathrm{MeV}$. Because there are so many more photons than baryons, we need to wait until the amount of photons in the tail of the energy spectrum with $E>2.2 \mathrm{MeV}$ becomes smaller than the number of $\mathrm{D}$ nuclei, namely when $n_{\gamma} \exp (-2.2 \mathrm{MeV} / T) \lesssim n_{B}$. When this happens, at $T \simeq$ $2.2 \mathrm{MeV} / \ln \left(\eta^{-1}\right)$ the so called "D bottleneck" finishes and ${ }^{4} \mathrm{He}$ is formed. Because until the end of the "D-bottleneck" period neutrons continue decaying, a large value of $\eta$ leads to a shorter time for neutrons to decay what leads to larger amounts of ${ }^{4} \mathrm{He}$ produced. In order to understand the duration of this period it is necessary to take into account the reheating of photons due to $e^{+} e^{-}$annihilation (see section 5) that happens precisely in this period. Due to this effect the temperature decreases less rapidly and the "D-bottleneck" finishes at $t \simeq 200 \mathrm{sec}$ (without reheating it would finish at $t \simeq 7.35 \mathrm{sec}$ instead).

At this point practically all neutrons end up in ${ }^{4} \mathrm{He}$ thus $n\left({ }^{4} \mathrm{He}\right)=n_{n} / 2$. Using $n_{n} / n_{p} \simeq 1 / 7$ we obtain with a back-of-an-envelope calculation the right amount of ${ }^{4} \mathrm{He}, Y\left({ }^{4} \mathrm{He}\right)=0.25$ :

$$
Y\left({ }^{4} \mathrm{He}\right)=\frac{4 n\left({ }^{4} \mathrm{He}\right) m_{p}}{\left[4 n\left({ }^{4} \mathrm{He}\right)+n(\mathrm{H})\right] m_{p}}=\frac{4\left(n_{n} / 2\right)}{4\left(n_{n} / 2\right)+n_{p}-n_{n}}=\frac{2\left(n_{n} / n_{p}\right)}{1+\left(n_{n} / n_{p}\right)}
$$

that is precisely 0.25 . Here $n\left({ }^{4} \mathrm{He}\right), n(\mathrm{H})$ are the number densities of ${ }^{4} \mathrm{He}$ and $\mathrm{H}$ nuclei and $m_{p}$ stands for the mass of both, protons and neutrons.

Trace amounts of ${ }^{7} \mathrm{Li}$ are also produced, but not heavier elements. The elements with atomic weight 5 and 8 are not stable and the gap in mass is then too large for heavier elements to be formed. This gap is bridged in stars at higher $T$ though threebody collisions. There are two different processes through which ${ }^{7} \mathrm{Li}$ is produced, that cross in a dip at $\eta \simeq 3 \times 10^{-10}$.

In addition, some amount of $\mathrm{D}$ and ${ }^{3} \mathrm{He}$ are left unburnt, as the reactions that burn them into ${ }^{4} \mathrm{He}$ freeze-out. The rates of these reactions are proportional to $\eta$. So for larger values of $\eta$ the reactions that burn D and ${ }^{3} \mathrm{He}$ are longer in equilibrium and less $\mathrm{D}$ and ${ }^{3} \mathrm{He}$ remain.

A range in $\eta$ is translated into a range of $\Omega_{B} h^{2}$, since $\eta=\left(n_{B} / n_{\gamma}\right)=$ $\left(\Omega_{B} \rho_{c} / m_{p}\right) /\left(2 \xi(3) T^{3} / \pi^{2}\right)$. With $T=2.73^{\circ} K$ one obtains $\eta=272.2 \times 10^{-10} \Omega_{B} h^{2}$. Bounds on $\eta$ have changed over time, mainly due to observational uncertainties and different treatments of the errors. The ranges of the "Chicago group" have changed from $\eta=(4-7) 10^{-10}$, i.e. $0.015 \leq \Omega_{B} h^{2} \leq 0.026$ (and $N_{\nu}<3.4$ ) in 
1980's, to $\eta=(2.8-4) 10^{-10}$, i.e. $0.012 \leq \Omega_{B} h^{2} \leq 0.015$ (and $N_{\nu}<3.3$ ) in 1991, to $\eta=(2-6.5) 10^{-10}$, i.e. $0.007 \leq \Omega_{B} h^{2} \leq 0.024$ (and $N_{\nu}<3.9$ at $95 \%$ C.L.) in 1995 [28]. The newer "Ohio group" prefers $\eta=(3.8-5.2) 10^{-10}$, i.e. $0.014 \leq \Omega_{B} h^{2} \leq 0.019$ (and $N_{\nu}<2.6$ at $95 \%$ C.L. as mentioned above) [26].

As a final comment, let us mention a particularly intriguing explanation for the result $N_{\nu}<3$, if confirmed. The solution may be an unstable $\nu_{\tau}$ with mass in the $\mathrm{MeV}$ range decaying with a lifetime $\tau<10$ sec into invisible particles [29], i.e. $\nu$ 's or $\nu \phi$ with $\phi$ a Goldstone boson, a Majoron. In particular if the daughter particles include $\nu_{e}$ and $m_{\nu_{\tau}}=1-10 \mathrm{MeV}$, these extra $\nu_{e}$ would deplete $n_{n}$ due to the process $\nu_{e} n \rightarrow p e^{-}$, because the opposite reaction $\bar{\nu}_{e} p \rightarrow n e^{+}$requires an energy of the daughter neutrinos $E_{\bar{\nu}_{e}}=m_{\nu_{\tau}} / 2>m_{n}-m_{p} \simeq \mathrm{MeV}$ and is suppressed for $m_{\nu_{\tau}}<10 \mathrm{MeV}$. This effect can be played off against the increase in $n_{n}$ (and consequent increase in ${ }^{4} \mathrm{He}$ ) due to extra contributions to the density $\rho$ during the BBNS to allow even for $N_{\nu}=16$ !

\section{Structure Formation}

The Universe looks lumpy at scales $\lambda \simeq 100 \mathrm{Mpc}$, we see galaxies, clusters, superclusters, voids, walls. But it was very smooth at the surface of last scattering of the CMBR (i.e. at electromagnetic decoupling, when ions and electrons first formed atoms) and later (see section 3). Inhomogeneities have been seen as anisotropies in the $\mathrm{CMBR}$, so $(\delta \rho / \rho)$ cannot be much larger than $\delta T / T \lesssim 10^{-5}$. So inhomogeneities in density start small and grow through the Jeans (or gravitational) instability: gravitation tends to further empty underdense regions and to further increase the density of overdense regions. One can follow analytically the evolution due to gravity of the density contrast $\delta \rho \equiv(\rho(x)-\rho) / \rho$ (where $\rho$ is the average density) in the linear regime where $\delta \rho / \rho<1$. In a static fluid the rate of growth of $\delta \rho / \rho$ is exponential, but in the Universe (an expanding fluid) it slows down into either a power law, $\delta \rho / \rho \sim a$, in a matter dominated Universe or it stops, $\delta \rho / \rho \simeq$ constant, in a radiation or a curvature dominated Universe. We have not so far mentioned the possibility of a Universe where the curvature term dominates the r.h.s. of Eq. (6). Using this equation one can see that a matter dominated open Universe becomes curvature dominated for $a \geq \Omega_{o} /\left(1-\Omega_{o}\right)$ and no further growth of the contrast of perturbations in matter can occur.

Perturbations have different physical linear dimensions $\lambda$ which increase with the Hubble expansion, $\lambda=a(t) \lambda_{\text {com }}$. Here $a(t)$ is the scale factor and $\lambda_{\text {com }}$ is the linear dimension measured in comoving coordinates (those that expand with the Hubble flow). With the usual choice of $a=1$ at present, $\lambda_{\text {com }}$ is the present actual linear dimension. Since $a \sim t^{\alpha}$ with $\alpha<1$ (see Eq. (8)) while the horizon $c t$ grows linearly with $t$, the horizon increases with time even in comoving coordinates, encompassing more material as time goes. When $\lambda=c t$ we say the perturbation of size $\lambda$ "enters" into the horizon, we could better say that the perturbation is first encompassed by the horizon. This moment is called "horizon-crossing" and it happens at different 
times for different linear scales $\lambda$, larger scales cross later.

What is the origin of these primordial fluctuations? In the standard BB model perturbations can at most cross the horizon only once, coming from outside the horizon, where they cannot be generated because there can be no causal interactions. In inflationary models each perturbation crosses the horizon twice. Inhomogeneities in density are generated as quantum fluctuations, expand exponentially going outside the horizon during inflation and re-enter later, after inflation is over. Inhomogeneities could also be generated within the horizon by defects, such as strings and textures, that are remnants from phase transitions in the early Universe.

It is convenient to specify the spectrum of fluctuations at horizon-crossing, $(\delta \rho / \rho)_{\text {hor }}$. A Harrison-Zel'dovich spectrum is scale invariant at horizon-crossing, namely $(\delta \rho / \rho)_{\text {hor }}=$ constant. COBE observations have shown this spectrum is in fact scale invariant or very close to it.

After horizon-crossing, physical interactions act upon the inhomogeneities and generate a "processed" spectrum. Three main processes compete. Gravitational interactions tend to produce a gravitational collapse, pressure changes tend to produce pressure waves, and there may be damping, caused by the free streaming of non-interacting particles (such as relativistic neutrinos) or due to collisions (this is the case of the Silk damping for baryonic matter, due to photon viscosity). A gravitational collapse only occurs if the distance a pressure wave travels before the collapse, $v_{\text {sound }} t_{\text {free-fall }} \equiv \lambda_{\text {Jeans }}$, is smaller than the size $\lambda$ of the inhomogeneity, i.e. $\lambda>\lambda_{\text {Jeans }}$. If so, roughly speaking, pressure waves cannot scape from the collapsing inhomogeneity. This is Jeans criterium for a collapse.

For baryons before recombination $v_{\text {sound }} \simeq c / \sqrt{3}$ (since $v_{\text {sound }}^{2}=d p / d \rho$ and the pressure is due to photons, thus $p=\rho / 3$ ), thus the Jeans length is larger than the horizon and the density contrast $(\delta \rho / \rho)_{B}$ of perturbations in baryons within the horizon cannot grow. After recombination, matter (atoms) decouple from radiation (since atoms are neutral), the pressure is due to non-relativistic $\mathrm{H}$ atoms thus the sound speed and consequently the Jeans length decrease steeply and perturbations in baryons can star growing. Moreover, Silk damping at decoupling erases inhomogeneities in baryons smaller than those corresponding to clusters of galaxies.

We can now see that structure could not have been formed with baryons alone. Because the Universe has become matter dominated at $t_{\text {eq }}$ before recombination, $t_{\text {eq }}<t_{\text {rec }}$, perturbations in baryons can grow as $(\delta \rho / \rho)_{B} \sim a$ from recombination onwards. However, the maximum growth factor since then is $a_{o} / a_{\text {rec }} \approx 10^{3}$, and with $(\delta \rho / \rho)_{\text {rec }}<10^{-4}$, as shown by COBE and other CMBR anisotropy experiments, $(\delta \rho / \rho)_{B}$ at present could not have reached 1. However, collapsed objects (galaxies, clusters etc.) have $\delta \rho / \rho>1$. Therefore, we conclude that there is not enough time from recombination to the present for inhomogeneities in baryons to grow to $(\delta \rho / \rho)_{B} \simeq 1$ (after that, in the "non-linear" regime, $\delta \rho / \rho$ grows very fast) unless baryons after recombination (i.e. when finally they can collapse into structures) fall into already existing potential wells. These potential wells can only be formed by matter not coupled to photons, namely dark matter (DM). Perturbations in the DM can start growing before recombination, as soon as the Universe becomes 
matter dominated (by the DM itself), so they have enough time to become non linear by now. By recombination the DM has formed the potential wells into which baryons fall. The necessity of a matter component in which density perturbations at the moment of recombination may be much larger than the perturbations in baryons, is a strong motivation for the existence of DM.

The question of which structures are formed first leads to the distinction of three types of DM: hot, warm and cold. The following naive picture of how fluctuations evolve helps understanding the differences of these three types.

Let us define as $t_{\text {gal }}$, the moment at which the growing horizon encompasses for the first time a perturbation of the size of a typical galaxy that contains, $10^{11} \mathrm{M}_{\odot}$ (including the dark halo), where $\mathrm{M}_{\odot}$ is the mass of the Sun. The temperature of the Universe at that moment turns out to be $T \simeq 1 \mathrm{keV}$. Hot DM particles are still relativistic at $t_{\text {gal }}$, i.e. their mass is $m<1 \mathrm{keV}$. In this case, at $t_{\text {gal }}$ each of the particles in a fluctuation of the size of the galaxy has moved from its original position a distance $c t$ equal to the size of the fluctuation. The volume occupied by the particles will expand with the horizon. Thus, a moment later the fluctuation is erased. This is damping by free streaming. At the moment when the DM particles become non-relativistic, their motion becomes negligible. Thus the smallest structure that can survive the "free streaming" of relativistic particles and grow, is that one encompassed by the horizon when the particles are becoming non-relativistic. Light neutrinos are hot DM and the smallest structure that can survive contains the Jeans mass, $M_{\text {Jeans }}=3 \times 10^{15} M_{\odot} /\left(m_{\nu} / 30 \mathrm{eV}\right)^{2}$ that corresponds to a supercluster. Therefore, with hot DM (HDM) (such as light neutrinos of $m \lesssim 30 \mathrm{eV}$ ) superclusters of galaxies form first and galaxies must form later, through the fragmentation of the larger structures. Simulations have shown, however, that there is not enough time to form galaxies. Thus HDM is rejected as the dominant DM component. Particles of mass $m \simeq \mathrm{keV}$, that are just becoming non-relativistic at $t_{\text {gal }}$, are warm DM. With warm DM galaxies form first, but barely. Cold DM (CDM) is such that perturbations smaller than a galaxy, even of the size of the smallest dwarf galaxy $\left(10^{6} \mathrm{M}_{\odot}\right)$, are not erased and can grow.

Simulations have shown that CDM must be the most abundant form of matter, because the "processed" spectrum of perturbations (i.e. the present spectrum once the effect of physics acting within the horizon has been considered) generated in standard CDM models reproduces the observations within 10\%. Standard CDM models, make the simplest assumptions, namely $\Omega_{\mathrm{CDM}}+\Omega_{B} \simeq \Omega=1, \Lambda=0$, scale invariant perturbations at horizon crossing, and a scale independent "biasing" by which only the highest peaks in the CDM density distribution end up forming galaxies. There is only one feature in the processed spectrum of CDM perturbations, a change of slope at the present scale that corresponds to the horizon at the moment of matter-radiation equality, $\lambda_{\text {eq }}$. Perturbations with $\lambda<\lambda_{\text {eq }}$ entered into the horizon at $t<t_{\mathrm{eq}}$, when the Universe is radiation dominated. They cannot grow while the Universe is radiation dominated, so they all start growing together at $t=t_{\text {eq }}$ and they roughly have the same amplitude today if they all started with the same amplitude at horizon crossing. Perturbations with $\lambda>\lambda_{\text {eq }}$, instead, en- 
tered into the horizon at $t>t_{\mathrm{eq}}$, when the Universe is matter dominated, so they started growing immediately. Consequently, perturbations at larger scales entered later, had less time to grow and their amplitude is smaller at present. Once $\lambda_{\text {eq }}$ (the location of the change slope) is fixed, the only remaining free parameter is an overall normalization of the CDM spectrum, now provided by the CMBR anisotropy measured by COBE at large scales, $\theta>20^{\circ}$. Density perturbations at these large scales entered into the horizon very recently (so they did not grow much), thus providing a measurement of $(\delta \rho / \rho)$ at horizon crossing, $(\delta \rho / \rho)_{\text {hor }}$. For more details on this section so far see e.g. [5].

While both the shape and normalization so obtained are almost right, they do not fit the observations [30]. In Fig. 4 [31] the power spectrum of density perturbations predicted by standard CDM models (solid line) normalized by COBE data (the box on the l.h.s.) and other data (the points with vertical error bars) are shown. As can be seen in the Fig. 4 (solid line) the spectrum of standard CDM models has too much power on small scales (large $\mathrm{k} \sim \lambda^{-1}$ ), the scales of galaxy clusters and smaller. Also in Fig. 4 one can see the failure of HDM to account for the data (see the short-dashed curve) mentioned above.

Once the normalization given by COBE is fixed, there are several possibilities to change the spectrum to agree with observations. Because HDM tends to erase structure at small scales, one of the solutions consists in adding to the CDM a bit of HDM, namely neutrinos, in what are called mixed DM (MDM) or hot-cold DM (HCDM) models [32]. See the dotted line in Fig. 4. In particular, models with $\Omega_{\nu}=0.2$, what corresponds to $\sum_{i} m_{\nu_{i}}=5 \mathrm{eV}$, and the rest of $\Omega_{o}$ in CDM plus some baryons, with $\Omega=1$ and $\Lambda=0$ works well. Another possibility is that of a "tilted" primordial spectrum of fluctuations at horizon crossing, one that slightly favors larger scales over smaller scales (instead of the flat, scale invariant, HarrisonZel'dovich spectrum) within the COBE observational limits. This is called "tilted"

FIGURE 4. Comparison of the measured power spectrum of density perturbations and the predictions of several DM models (notice that $\mathrm{k} \sim \lambda^{-1}$ ). 
CDM (TCDM) [33]. See the long-dashed line (labelled with $\mathrm{n=0.8)}$ ) in Fig. 4.

Another family of solutions is obtained by realizing that a shift towards larger scales of the only feature in the CDM spectrum, i.e. $\lambda_{\text {eq }}$, the scale where the slope changes, is enough to provide good agreement with observations, since it effectively amounts to increasing the power of the spectrum at scales larger than the break point $\left(\lambda>\lambda_{\text {eq }}\right)$ with respect to those smaller than it $\left(\lambda<\lambda_{\text {eq }}\right)$. The break in the slope occurs at the moment of radiation-matter equality, $\rho_{\text {rad }}=\rho_{\text {matter }}$, when the Universe becomes matter dominated (by the DM). The scale of the break, $\lambda_{\text {eq }}$, corresponds to the present size of the horizon at matter radiation equality, $c t_{\mathrm{eq}}$. It is $\lambda_{\text {eq }} \simeq 10 \mathrm{Mpc}\left[\left(g_{*} / 3.36\right)^{1 / 2}\left(\Omega_{o} h^{2}\right)^{-1}\right]$, where $\Omega_{o}$ is essentially the present matter density, since $\Omega_{R}<<\Omega_{o}$ (recall that the total density is $\Omega=\Omega_{o}+\Omega_{\mathrm{vac}}$, if the cosmological constant $\Lambda \neq 0$ ) and $g_{*}$ is the effective number of relativistic degrees of freedom at $t_{\text {eq }}, g_{*}=3.36$ for photons plus three relativistic neutrino species (see section 5).

The equation $\lambda_{\text {eq }} \equiv\left(10 h^{-1} M p c\right) \Gamma^{-1}$ defines the "shape parameter" [34] $\Gamma \equiv$ $\Omega_{o} h\left(g_{*} / 3.36\right)^{-1 / 2}$ (notice that distances determined through red-shifts are given in $h^{-1} M p c$ units (see Eq. (5)). The data require $\Gamma \simeq 0.25 \pm 0.05$, while standard CDM models (with the standard choices of $h=0.5, \Omega_{o}=1, g_{*}=3.36$ ), has $\Gamma=0.5$. In fact, as we have explained a larger $\lambda_{\text {eq }}$, thus a smaller $\Gamma$, would provide agreement with data. In order to lower the value of $\Gamma$ with respect to that of the standard CDM model one needs to either: 1- lower $h$ [35] (what implies an older Universe), or 2- increase $g_{*}$ (namely increase the radiation content of the Universe at $t_{\text {eq }}$ ), or 3- lower $\Omega_{o}$ (i.e. take $\Omega_{o}<1$ ), so that we either live in an open Universe (open CDM models, OCDM) if $\Lambda=0$, or in a Universe with a cosmological constant that provides $\Omega_{\mathrm{vac}}=1-\Omega_{o}\left(\Lambda \mathrm{CDM}\right.$ models [36]), for example with $h=1, \Omega_{\mathrm{CDM}}=0.18$, $\Omega_{B}=0.02, \Omega_{\mathrm{vac}}=0.80$, or 4 - a combination of all three above. We have already examine the consequences of the $3 \mathrm{rd}$. solution in relation with the possible "age crisis", in section 3.6. Let us examine the consequences of the other two.

If we want to keep $\Omega_{o}=1$, with the standard value of $g_{*}=3.36$ one would need $h=0.3$ to get $\Gamma=0.3$, but this would lead to a very old Universe with $t_{o}=2.2 \times 10^{10} \mathrm{yr}$. If instead we keep the standard value of $h$ i.e. $h=0.5$ (this low-value of $h$ is necessary with $\Omega_{o}=1$ to account for $t_{o}=1.3 \times 10^{10} \mathrm{yr}$ ), the value $g_{*}=9.33$ is necessary to get $\Gamma=0.3$, what amounts to the equivalent of 16 relativistic neutrino species. However, primordial nucleosynthesis does not allow for much more than 3 neutrino species (except with a heavy unstable $\nu_{\tau}$, see section 6 and below).

A way to obtain the large amount of radiation needed is through a heavy neutrino decaying into radiation, with the right combination of mass and lifetime, in so-called $\tau$ CDM models [37] [38]. A massive neutrino matter dominates the energy density of the Universe as soon as it becomes non-relativistic $m_{\nu} \gtrsim T$ ( since $n_{\nu} \simeq n_{\gamma}$ and $\left.\rho_{\nu}=n_{\nu} m_{\nu}, \rho_{\text {rad }} \simeq n_{\gamma} T\right)$, thus their decay products radiation-dominate the Universe at decay. For $m_{\nu}<1 \mathrm{MeV}$ the right mass-lifetime combination lie on a narrow strip around the (usually called) "galaxy formation" bound. This bound [39] is obtained 
by requiring that the decay products of a massive neutrino cease to dominate the energy density of the Universe before baryons should start falling into gravitational wells, at recombination $t_{r e c} \simeq 10^{-5} t_{o}$, what yields $\tau \leq\left(92 \mathrm{eV} / m_{\nu}\right)^{2}\left(\Omega_{o} h^{2}\right)^{2} 10^{-5} t_{o}$. Near this bound, at the boundary between being irrelevant and harmful, unstable neutrinos could help in the formation of structure in the Universe. A heavier neutrino, of $m_{\nu} \simeq 1-10 \mathrm{MeV}$, necessarily $\nu_{\tau}$, decaying at or just before nucleosynthesis, $\tau=0.1-100 \mathrm{sec}$, would also provide a solution [38]. If $\tau=0.1$-few sec and $\nu_{e}$ are among the decay products, neutrons would be depleted, leaving room for the presence of up to 16 equivalent relativistic neutrino species during nucleosynthesis [29], as explained at the end of section 6 . This $\nu_{\tau}$ decays too fast to get to matter dominate so the decay products never dominate the energy density of the Universe and additional unknown radiation has to be added ad-hoc to increase $g_{*}$ as needed. If instead $\tau_{\nu} \simeq 10-100 \mathrm{sec}$, the $\nu_{\tau}$ can dominate the energy density of the Universe before decaying and its decay products provide the necessary extra radiation. The $\nu_{\tau}$ decay modes involved here should all be into neutral particles, $\nu_{\tau} \rightarrow 3 \nu^{\prime} \mathrm{s}$ or $\nu_{\tau} \rightarrow \nu \phi$, with $\phi$ a Majoron (a zero mass Goldstone boson) for example. All visible modes, i.e. producing electrons or photons, are forbidden in the necessary range.

All these modified CDM models seem to be able to fit present data, however they predict very different patterns of acoustic peaks in the CMBR anisotropy power spectrum $\ell(\ell+1) C_{\ell}$ (see section 3.3 and Eq. (10)). The location and relative height of the peaks between $\ell \simeq 200$ and several 1000 characterize different models. The detection of the CMBR anisotropies so far obtained with the COBE satellite and balloon experiments, does not discriminate between models, but the next generation of observations will do it. There are two approved new satellite experiment with sub-degree resolution, MAP in the U.S. and COBRAS/SAMBA in Europe. They will give results as shown in the Fig. 2 [13] together with the predictions of four different models, three of them mentioned above SCDM (standard CDM), $\Lambda$ CDM (CDM with $\Lambda>0$ ) and OCDM (open CDM), and one labelled with "strings", that corresponds to structure formation due to inhomogeneities caused in a phase transition that produced "cosmic strings" (vortex-like solitonic remnants). The dark band around the SCDM line shows the expected experimental error in the $C_{\ell}$ 's for an experiment like COBRAS/SAMBA, with $10^{\prime}$ beam-size and $10 \mu \mathrm{K}$ noise (that would allow to resolve anisotropies up to $\sim 0.5^{\circ}$ for $\Omega=1$ ) [13]. It is clear from Fig. 2 that the different theoretical models shown could be easily distinguished.

\section{Detection of the Dark Matter in the Halo of Our Galaxy}

Our own galaxy is spiral. The Sun is part of a thin disk of about $12 \mathrm{kpc}$ of radius consisting mainly of stars and gas clouds orbiting the galactic center. The Sun is at $r_{o} \simeq 8.5 \mathrm{kpc}$ from the center and moving at about $v_{c} \simeq 220 \mathrm{~km} / \mathrm{sec}$. The rotation curve of the Milky Way is flat at about $v_{c}$ out to as far as it is measured, showing that our galaxy has a spherical or ellipsoidal dark halo. Assuming a 
spherical and isotropic velocity distribution, the density profile of this halo is usually parametrized as $\rho(r)=\rho_{o}\left[\left(a^{2}+r_{o}^{2}\right) /\left(a^{2}+r^{2}\right)\right]$ where $\rho_{o} \simeq 0.01 M_{\odot} / \mathrm{pc}^{3} \simeq 0.4$ $\mathrm{GeV} / \mathrm{cm}^{3}$ is the halo density in the solar neighbourhood and $a$ is the core radius of the halo $(\rho \simeq$ const at $r<a$ ), that has been estimated at $a \simeq 2-10 \mathrm{kpc}$. There is also a central luminous component of the galaxy, the spheroid or bulge. The distribution and motion of the luminous matter trace the combined gravitational effects of the three components, the disk, the bulge and the halo. The halo dominates at large radius.

In the last ten years, attempts have been made to detect the galactic DM in experiments that could reveal its nature. Because the DM in galactic haloes amount to $\Omega_{\text {haloes }} \simeq 0.02 h^{-1}$ (see section 3.7 ) nucleosynthesis bounds on the baryonic density $\Omega_{B}$ (see section 6 ) do not preclude the possibility that "dark" baryons constitute some or all the DM in galaxy haloes like ours. Candidates for dark baryons could be macroscopic objects up to the size of "brown dwarfs". These are stars too small, $m<0.08 M_{\odot}$, for their centers to reach a sufficiently high temperature to burn hydrogen (as in a normal star). These candidates are called MACHOs (for massive compact halo objects) as opposed to WIMPs (for weakly interacting massive particles), that are elementary particle candidates for the DM. Elementary particle candidates include also massive neutrinos (actually the only DM particle candidates known to exist, but not known to be massive as needed). The three known neutrinos, if stable, are hot DM candidates, and the energy they would carry is too small to allow their detection in the dark halo of our galaxy (but the measurement of their masses in the range 1 -few $10 \mathrm{eV}$ could tell). The theoretically preferred cold DM candidates are WIMPs, with masses in the range $1 \mathrm{GeV}-1 \mathrm{TeV}$, and the axion, a very light boson that appears in the Peccei-Quinn solution to the strong $\mathrm{CP}$ problem of elementary particles, with a mass $m \simeq 10^{-5} \mathrm{eV}$.

WIMPs appear in many extensions of the standard model of elementary particles, such as the minimal supersymmetric extension of the standard model, where the usual WIMP candidate is the lightest "neutralino" (the lightest fermionic partner of the neutral gauge and Higgs bosons). As we have seen (see Eq. (12)), it is enough for a particle in the $\mathrm{GeV}-\mathrm{TeV}$ mass range to have annihilation cross sections of weak order in order to have a relic abundance $\Omega \simeq 0(1)$. WIMPs are being search for in direct and indirect DM searches and accelerator experiments. If these particles compose the halo of our galaxy they may be detected directly, through the energy they may deposit in collisions with nuclei within detectors, or indirectly through their annihilation products, coming from annihilations in the Sun or Earth, where they may accumulate, or in the halo itself. Direct WIMP searches look for energies of order $m v^{2} \simeq 0(10 \mathrm{keV})$ deposited in ionization and phonons, in $\mathrm{kg}$ size cryogenic crystals, or producing scintillation or excitations of superfluids and superconductors. Indirect WIMP detectors search mainly for high energy neutrinos produced in WIMP annihilations in the Sun and the Earth, in large water or ice Cerenkov light detectors (such as Superkamiokande, AMANDA, DUMAND and NESTOR) or underground detectors (such as MACRO). For a review of particle DM searches see e.g. [40]. 
Axions could be detected through their coupling to two photons, looking for the resonant conversion of halo axions to microwave photons in a cavity with a magnetic field. The first detector of this type capable to actually test halo axions is based at Livermore [41] and has started taking data recently.

The most fruitful DM searches so far, have been those looking for MACHOs though the gravitational microlensing [42] of background stars in the Large Magellanic Cloud (LMC), an irregular galaxy satellite of the Milky Way. In a gravitational lensing event an intervening object located in the line of sight of a luminous object deflects the light coming from it, producing multiple images. In a microlensing event, these multiple images are superposed, what is seen as an increase in the brightness of the lensed object. Three independent groups the American-Australian MACHO, the French EROS and the Polish-American OGLE collaborations (that seem to be competing for the most politically incorrect name) have seen microlensing events. They were looking for (and found in 1993 [43]) events in which a star from the LMC or the bulge of our galaxy symmetrically brightens and fades, as a MACHO (or a not seen faint star) passes near its line of sight (one could describe the event as a symmetric "anti-eclipse") during a time $\Delta t$ that depends on the lensing mass $M, \Delta t=140$ days $\left(M / M_{\odot}\right)^{1 / 2}$. So far about 90 events have been seen in the direction of the central bulge of our galaxy (where most lenses are expected to be faint stars in the bulge itself) and about 10 towards the LMC. These numbers are about a factor of 5 too small from what was expected from the LMC if the dark halo of the Milky Way consisted of MACHOs and about a factor of 3 too large from what was expected from the bulge (see e.g. [44]).

The number of events towards the LMC is too small to be compatible with a dark halo dominated by MACHOs (within the mass range tested $M \simeq 10^{-7}-1 M_{\odot}$ ). Machos could account for up to $\sim 30 \%$ of the expected halo mass, unless the halo is much lighter than presently believed [45]. The large number of microlensing events towards the bulge, where the lensing objects are expected to be mostly faint stars, is much larger than expected on the basis of assuming a spherical bulge in the center of our galaxy, as seen in regular disk galaxies. The excess indicates that the bulge of our galaxy is a bar with its axis oriented towards the Sun [46], in agreement with other observations, what would yield a larger star density along our line of sight towards the bar. An alternative explanation could be that the mass of the disk is much larger than in standard galactic models. More data will allow to distinguish these possibilities.

\section{Acknowledgments}

I thank the organizers of this workshop for their invitation. This work was supported in part by the U.S. Department of Energy under Grant DE-FG03-91ER 40662 TaskC. 


\section{REFERENCES}

1. G. Gamow, Phys. Rev. 70, 572 (1946). R. Alpher, H. Bethe and G. Gamow, Phys. Rev. 73, 803 (1948).

2. R. A. Alpher and R.C. Herman, Phys. Rev. 75. 1089 (1949).

3. A. Penzias and R. Wilson, Ap. J. 142, 419 (1965).

4. R.H. Dicke et al. Ap. J. 142, 414 (1965).

5. W. Kolb and M. Turner, The Early Universe, Addison-Wesley, 1990.

6. J.A. Frieman, Fermilab-Conf.95/151-A, Proc. Snowmass '94 "Particle and Nuclear Astrophysics and Cosmology in the Next Millenium", eds. E. Kolb and R. Peccei, World Scientific (1995).

7. (CfA Survey) V. de Lapparent, M. Geller and J.P. Huchra Ap. J. Lett. 302, L1 (1986); M. Geller and J.P. Huchra, Science 146, 897 (1989).

8. D. Lynden Bell et al. Ap. J. 326, 19 (1988)

9. A. Deckel, astro-ph/9401022, Ann. Rev. Astron. Astrophys. 32 (1994).

10. (COBE Coll.) G.F. Smoot et al. Ap. J. Lett. 396, L1 (1992); (COBE Coll.) C. L. Bennet et al. Ap. J. 436, 423 (1994).

11. M. White, D. Scott and J. Silk, Ann. Rev. Astron. Astrophys. 32, 319 (1994); D. Scott, J. Silk and M. White, Science 268, 829, 1995; W. Hu , N. Sugiyama, J. Silk, IASSNS-AST-96-30, astro-ph/9604166, to appear in Nature.

12. (COBE Coll.) J.C. Mather et al. Ap. J. 420, 439 (1994).

13. D. Scott, CFPA-95-TH-51, astro-ph/9509035.

14. W. Freedman et al. Nature 371, 757 (1994).

15. J. Cowan, F. Thielemann and J. Truran, Ann. Rev. Astron. Astroph. 29, 447(1991).

16. D. Winget et al. Ap. J. Lett. 315, L77 (1987); I. Iben and G. Laughlin, Ap. J. 341, 430 (1989).

17. A. Renzini Proc. 16th Texas Symp. on Relat Astroph. and 3rd Symp. on Particles, Strings and Cosmol. eds. C. Akerlof and M. Srednicki, N.Y. Acc. of Sci., N.Y. (1992); X. Shi Ap. J. (1995).

18. M. Fukugita and E. Turner, Monthly Not. of the Royal Ast. Soc. 253, 99 (1991); C. Kochanek Ap. J. 419, 12 (1994), D. Maoz and H. Rix, Ap. J. 416, 425 (1994).

19. V. Trimble, Ann. Rev. Astron. Astrophys. 25, 425 (1987).

20. S. Faber and J. Gallagher, Ann. Rev. Astron. Astrophys. 17, 135 (1979).

21. R. Lynds and V. Petrosian, Bull. American Astron. Soc., 18, 1014 (1986); G. Soucail et al. Astron. and Astrophys. 172, L14 (1987); J. Tyson, F. Valdes and R. Wenk, Ap. J. Lett. 349, L19 (1990).

22. M. White, J. Navarro, A. Evrard and C. Frenk, Nature 366, 429 (1993).

23. G. Steigman and J. Felten, OSU-TA-24-94 astro-ph/9502029.

24. G. Gerstein and Ya B. Zel'dovich, Zh. Ekspt. Teor. Fiz. Pis'ma Red. 4, 174 (1996) (and others, see e.g. [5]).

25. B. W. Lee and S. Weinberg, Phys. Rev. Lett. 39, 165 (1977) (and others, see e.g. $[5])$.

26. N. Hata et al., Phys.Rev.Lett. 75, 3977 (1995).

27. C. Cardall and G. Fuller, UCSD preprint, astro-ph/9603071.

28. J. Yang et al., Ap. J. 281, 493 (1984); T. P. Walker et al. Ap. J. 376, 51 (1991), C. 
Copi, D. Schramm and M. Turner, Phys. Rev. Lett. 75, 3981 (1995).

29. S. Dodelson, G. Gyuk and M. Turner, Phys. Rev. D49, 5068 (1994); M. Kawasaki et al., Nucl. Phys. B419, 105 (1994).

30. J.P. Ostriker, Ann. Rev. Astron. Astrophys. 31, 689 (1993); A.D. Liddle and D. Lyth, Phys. Repts. 231, 1 (1993).

31. W. Kolb, Proc. 42nd Scottish School if Physics, Univ. of St. Andrews, Scottland (1993) and Fermilab-Conf-94/058-A.

32. Q. Shafi and F. Stecker, Phys.. Rev. Lett. 53, 1292 (1984); C.P. Ma and E. Bertschinger, Ap. J. 429, 22 (1994) and Ap. J. 434, L5 (1994), J. Primak et al., Phys.. Rev. Lett. 74, 2160 (1995).

33. R. Cen et al. Ap. J. 399, L11 (1992), R. Davis et al.Phys. Rev. Lett. 69, 1856 (1992); F. Lucchin, S. Mattarese and S. Mollerach, Ap. J. 401, L49 (1992).

34. G.E. Efstathiou, Proc. of the "36th Scottish Univs Summer Sch. in Phys", "Phys. of the Early Universe", ed. J. Peacock, A. Heavens and A. Davies Adam-Hilger, N.Y.(1990) p. 361; J. Peacock and S. Dodds. Monthly Not. of the Royal Ast. Soc. 267, 1020 (1994).

35. J. Bartlett et al., Science 267, 980 (1995).

36. M.S. Turner, G. Steigman and L. Krauss, Phys. Rev. Lett. 52, 2090 (1984); L. Krauss and M. Turner CWRV-P6-95, astro-ph/9504003.

37. J. Bardeen, J. Bond and G. Efstathiou, Ap. J. 321, 28 (1997); J. Bond and G. Efstathiou, Phys. Lett. B265, 245 (1991); M. White, G. Gelmini and J. Silk, Phys. Rev. D51, 2669 (1995).

38. S. Dodelson, G. Gyuk and M.S. Turner, Phys. Rev. Lett. 72, 3578 (1994).

39. P. Hut and S. White, Nature 310, 637 (1984); G. Steigman and M. Turner, Nucl. Phys. B 253, 375 (1985).

40. B. Sadoulet Proc. Snowmass '94 "Particle and Nuclear Astrophysics and Cosmology in the Next Millenium", eds. E. Kolb and R. Peccei, World Scientific (1995); K. Griest ibid.

41. K. van Bibber Proc. "Yth Meeting APS, DPF", eds. C. Albright, P. Kasper, R. Raja and J. Yoh, World Scientific, Singapore (1992).

42. B. Paczynski, Ap. J. 304, 1 (1986).

43. (MACHO Coll.) C. Alcock et al., Nature 365, 623 (1993); (EROS Coll.) E. Aubourg et al., Nature 365, 623 (1993); (OGLE Coll.) A. Udalski et al. Acta Astronomica 43, 289 (1993).

44. (MACHO Coll.) D. Bennett et al., astro-ph/9510104 B; Paczynski, astro$\mathrm{ph} / 9412049$.

45. E. Gates, G. Gyuk and M. Turner FERMILAB-Pub-95/090-A, astro-ph/9505039.

46. H. Zhao, D. Spergel and M. Rich, Ap. J. 440, L13 (1995); Paczynski B. et al., Ap. J. 435, L113 (1995). 